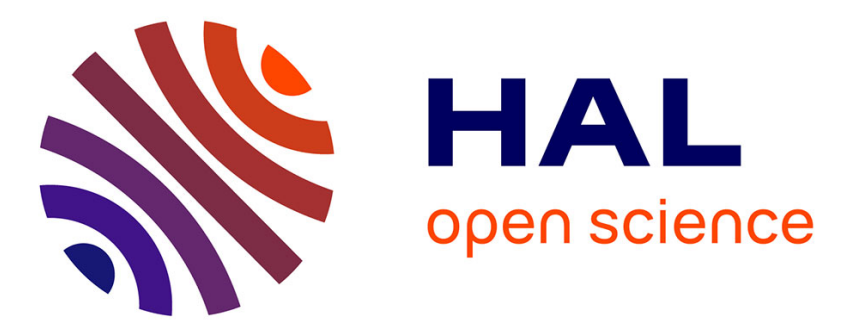

\title{
Variational formulation of pre-stressed solid-fluid mixture theory, with an application to wave phenomena
}

Luca Placidi, Francesco Dell'Isola, Nicoletta Ianiro, Giulio Sciarra

\section{To cite this version:}

Luca Placidi, Francesco Dell'Isola, Nicoletta Ianiro, Giulio Sciarra. Variational formulation of prestressed solid-fluid mixture theory, with an application to wave phenomena. European Journal of Mechanics - A/Solids, 2008, pp.25. hal-00499568

\section{HAL Id: hal-00499568 https://hal.science/hal-00499568}

Submitted on $10 \mathrm{Jul} 2010$

HAL is a multi-disciplinary open access archive for the deposit and dissemination of scientific research documents, whether they are published or not. The documents may come from teaching and research institutions in France or abroad, or from public or private research centers.
L'archive ouverte pluridisciplinaire HAL, est destinée au dépôt et à la diffusion de documents scientifiques de niveau recherche, publiés ou non, émanant des établissements d'enseignement et de recherche français ou étrangers, des laboratoires publics ou privés. 


\title{
Variational formulation of pre-stressed solid-fluid mixture theory, with an application to wave phenomena
}

\author{
Luca Placidi $^{\mathrm{a}, \mathrm{b}, *}$, Francesco Dell' Isola ${ }^{\mathrm{a}, \mathrm{b}}$, Nicoletta Ianiro ${ }^{\mathrm{c}}$, Giulio Sciarra ${ }^{\mathrm{b}, \mathrm{d}}$ \\ a Sapienza, Universitá di Roma, Dipartimento di Ingegneria Strutturale e Geotecnica, Via Eudossiana, 18, 00184 Roma, Italy \\ b Sapienza, Universitá di Roma, Laboratorio di Strutture e Materiali Intelligenti, Palazzo Caetani (Ala Nord), 04012 Cisterna di Latina (Lt), Italy \\ c Sapienza, Universitá di Roma, Dipartimento Metodi e Modelli Matematici per le Scienze Applicate, Via Scarpa, 16, 00161 Roma, Italy \\ d Sapienza, Universitá di Roma, Dipartimento di Ingegneria Chimica, Materiali, e Ambiente, Via Eudossiana, 18, 00184 Roma, Italy
}

\begin{abstract}
Fluid saturated porous media are modelled by the theory of mixtures and the placement maps of the solid and of the fluid are considered. The momentum balance equations are derived in the framework of a variational approach: We take an action functional and two families of variations and assume that the sum of the virtual work of the external forces and the variation of such an action along each variation are zero. Constitutive equations for the two Cauchy stress tensors and for the interaction force are derived taking into account a general state of pre-stress for the solid and for the fluid species. Governing equations are therefore formulated, however, for the sake of simplicity, only the case of pure initial pressure is further investigated. The propagation of bulk (transversal and longitudinal) waves and the influence of pre-stress are studied: In particular, stability analyses are carried out starting from dispersion relations and the role of pre-stress is investigated. Finally, a numerical example is established for a given state of pre-stress, deriving the phase velocities and the attenuation coefficients of transversal and longitudinal waves.
\end{abstract}

Keywords: Pre-stress; Mixture; Variational approach; Waves; Soil

\section{Introduction}

\subsection{Historical background}

Recently, an increasing interest has been paid to the development of mathematical models (see e.g. Wilmanski, 1996; Svendsen, 2001; Sciarra et al., 2003; Placidi and Hutter, 2006) aiming to describe all the phenomena occurring in physical heterogeneous systems constituted by more than one constituent. Such an interest finds its motivation in the efforts made by the researchers in engineering sciences to control complex systems such as pressurised salt caverns including reacting mixtures of fluids (Cosenza et al., 1999), soils which are susceptible to debris flows or landslides (Pudasaini et al., 2005), snow masses susceptible to snowslides and avalanches (Wang et al., 2004),

\footnotetext{
* Corresponding author.

E-mail address: luca.placidi@uniroma1.it (L. Placidi).
} 
moraines and basal sediment layers supporting ice sheets either in the alpine glaciers or in Antarctica (dell'Isola and Hutter, 1998a, 1998b), cartilages and bone tissues in living organisms (Cowin, 1999), bone-implant interfaces in biomedical applications.

The classical models, which are available in the literature (see, e.g., Terzaghi, 1925; Fillunger, 1936) and are exploited in engineering applications, contain simplifications which were useful in order to obtain treatable mathematical problems to be solved in the design process. A typical example is the consolidation model due to Terzaghi (1925), which supplies a powerful conceptual tool to forecast the behaviour of soils supporting civil constructions. A detailed description of the contribution of Terzaghi to Engineering Sciences, including the applicability range of his model, can be found in de Boer (1996). Biot, somehow, improved the Terzaghi model enlarging the possibility of a scientifically based design in Geotechnique and originating an entirely new research field. The efforts of Biot are witnessed by the papers (Biot, 1941, 1955, 1956a, 1956b; Biot and Willis, 1957; Biot, 1962). The Biot model of porous materials is focused on the description of the behaviour of the solid skeleton, regarding the effects of the fluid on the overall body by introducing a kind of microstructural parameter which resembles a temperature-like one. More precisely this means that an energy potential can be defined for the skeleton, which depends both on the solid strain tensor and on the microstructural parameter and that such a dependency parallels similar constitutive equations in thermomechanics. Typically the introduced microstructural parameter measures the porosity distribution in the overall body (volume density of the voids) or the local density of the fluid (see e.g., Coussy, 2004; Beak and Srinivasa, 2004). In this framework, the porous material is required to satisfy the saturation condition. In the language of continuum mechanics theories (see e.g. the books Truesdell and Toupin, 1960; Truesdell and Noll, 1965; Müller, 1985; Liu, 2002), the extra-field introduced by Biot is an hidden-variable, i.e., a variable to be determined adding to the balance equations suitable constitutive equations which relate this parameter with the strain tensor and the fluid pressure.

Apparently this approach does not guarantee a complete symmetry between the solid and the fluid constituent; as a matter of facts, the governing equations describe the balance of momentum for the overall body and for the pure fluid (Darcy Law). Conversely the classical Theory of Mixtures (see Fillunger, 1936; Truesdell, 1957a, 1957b; Bowen, 1980; de Boer, 1996; Hutter and Jönk, 2004) preserves the symmetric structure of solid and fluid momentum balances, where the solid-fluid bulk interactions appear. The mixture model is indeed completely consistent with the general framework of modelling superposed continua, the characteristic of which is the presence of non-vanishing zerothorder terms in the overall virtual power. Indeed a deduction of the Biot model from the theory of binary solid-fluid mixture can be found in Coussy et al. (1998): In this framework the Terzaghi equation can be easily obtained as an application to a particular one-dimensional problem.

To the best of our knowledge, in the models available in the literature, see e.g. Truesdell (1957a; 1957b), Bowen and Chen (1975), Bowen (1976; 1980; 1982), de Boer and Ehlers (1990), Bluhm et al. (1995), de Boer (1996; 1998), Svendsen (2001), the initial pressure of the saturating fluid to the solid matrix configuration is considered as small enough to be negligible. Only in Wilmanski (2000) the aforementioned initial pressure is explicitly taken into account, however neglecting its effect on the dependence of the deformation tensor on the stress tensor. Having in mind some special applications in geomechanics and in particular the design of pressurised salt caverns (Cosenza et al., 1999), the situation in which the initial pressure of saturating fluid is large enough to be able to originate some instability phenomena (see also Smeulders et al., 1992; Sciarra, 2001; Sciarra et al., 2001), is explicitly considered. As a matter of facts, it is well known that high pressures of confined fluids in sealed salt caverns may cause highly destructive phenomena.

The consideration of non-vanishing initial partial pressures of the fluid and solid constituents induces stability arguments to arise, even in the case of linear problems. Indeed the presence of this additional parameter modifies the strong ellipticity conditions which guarantee existence and uniqueness of the linear elasticity problem (see e.g. Fichera, 1972; Bedford and Stern, 1972; Iesan, 1994; Benallal and Comi, 2003). It is therefore a crucial issue the characterisation of the restriction to be imposed to such pre-stress in order to have assured well-posedness and stability. In the present paper some stability conditions are captured with a simple analysis of the dispersion relation following the methods developed in Foch and Ford (1970) in a general case and in Bowen and Chen (1975), Borrelli and Patria (1984), Garg and Neyfeh (1986), Batra and Bedford (1988), Nigmatulin and Gubaidullin (1992), Smeulders and Van Dongen (1997), Abellan and de Borst (2006) for wave propagation in porous media. Preliminaries and notations ends the first section of this paper. 
In Section 2, we develop a variational derivation of the field equations. The generalisation, from mono to multiphase theories, of the variational procedure is not straightforward and we followed methods available in the literature, see e.g. Bedford and Drumheller (1978; 1979), Batra et al. (1986), Batra and Bedford (1988), Gouin (1990), Gavrilyuk et al. (1998), Gavrilyuk and Gouin (1999), Sciarra et al. (2003), Gouin and Ruggeri (2003), Gavrilyuk (2005). Two distinct families of variations have been defined and the balance equations have been deduced. Special attention has been paid to the interpretation of boundary conditions, taking into account the definition of global stresses (see Rajagopal and Tao, 1995; Rajagopal et al., 1986; Beak and Srinivasa, 2004).

In Section 3 constitutive relations are assumed; as a consequence, the constitutive equations are written in a general fashion and in the respective linearised form.

In Section 4 the set of the final linear partial differential equations have been solved via plane waves. The general dispersion relation have been deduced and an analysis of transverse and longitudinal waves has been performed.

In Section 5 we analyse the dispersion relation and find the condition of non-explosion (or linear stability condition) of the considered mixture in the general case. This is an important part of the paper because we give conditions on the values of pre-stress that the mixture can support.

In Section 6 we analyse the positive definiteness condition of the internal energy and find that it can be deduced by the linear stability condition.

In Section 7 we give a numerical example finding out how monocromatic wave propagation is affected by pre-stress and solid-fluid coupling, this last being described by a proper term of the internal energy.

Section 8 is devoted to some conclusions and the appendices terminate the paper.

\subsection{Preliminary and notation}

Let $\mathcal{B}_{s}$ and $\mathcal{B}_{f}$ be the reference configurations of the solid and of the fluid constituents, respectively. The actual configuration will be denoted by $\mathcal{B}_{t}$. In the theory of mixtures the place $\mathbf{x} \in \mathcal{B}_{t}$ in the actual configuration is the image at the same time of a solid and a fluid material particle under suitable placement maps. The material particles are the elements $\mathbf{X}_{s} \in \mathcal{B}_{s}$ and $\mathbf{X}_{f} \in \mathcal{B}_{f}$ of the reference configurations of the solid and of the fluid, respectively (see e.g. Fig. 1). This means that there exist two functions $\chi_{s}(\cdot, t)$ and $\chi_{f}(\cdot, t)$, called the current placements of the solid and of the fluid, such that every $\mathbf{x} \in \mathcal{B}_{t}$ in the actual configuration is the image under the aforementioned maps of the material particles $\mathbf{X}_{s}$ and $\mathbf{X}_{f}$,

$$
\mathbf{x}=\chi_{s}\left(\mathbf{X}_{s}, t\right)=\chi_{f}\left(\mathbf{X}_{f}, t\right), \quad \forall \mathbf{X}_{s} \in \mathcal{B}_{s}, \forall \mathbf{X}_{f} \in \mathcal{B}_{f}, \forall t \in \mathbb{R},
$$

where $t$ is the time and the functions $\chi_{\alpha}(\cdot, t), \alpha=s, f$ are assumed invertible and differentiable as many times as necessary. Let the inverse function of the current placements be $\chi_{s}^{-1}(\cdot, t)$ and $\chi_{f}^{-1}(\cdot, t),{ }^{1}$ i.e.,

$$
\mathbf{X}_{s}=\hat{\chi}_{s}^{-1}(\mathbf{x}, t), \quad \mathbf{X}_{f}=\hat{\chi}_{f}^{-1}(\mathbf{x}, t), \quad \forall \mathbf{x} \in \mathcal{B}_{t}, \forall t \in \mathbb{R} .
$$

The information contained in $\chi_{s}$ and $\chi_{f}$ can also be represented by the current displacements $\mathbf{u}_{s}$ and $\mathbf{u}_{f}$, that are defined as follows,

$$
\mathbf{x}=\mathbf{X}_{s}+\mathbf{u}_{s}\left(\mathbf{X}_{s}, t\right)=\mathbf{X}_{f}+\mathbf{u}_{f}\left(\mathbf{X}_{f}, t\right) .
$$

Let us consider a general scalar, vector or tensor function, e.g. $A$; whether we have the pedices $A_{\alpha}, \alpha=s, f$, or the superscript $\hat{A}$ we will interpret the related functional dependence as the following rules prescribe, viz.,

$$
A_{s}\left(\mathbf{X}_{s}, t\right), \quad A_{f}\left(\mathbf{X}_{f}, t\right), \quad \hat{A}(\mathbf{x}, t),
$$

which means that $A_{\alpha}$ are Lagrangian fields defined over the reference configuration of the $\alpha$-th constituent respectively, whilst $\hat{A}$ is an Eulerian field. In classical continuum mechanics the meaning of partial and total time derivatives are well-defined. In a mixture theory these concepts need a certain generalisation. We define,

$$
\frac{d_{s}}{d t}, \quad \frac{d_{f}}{d t}, \quad \frac{\partial}{\partial t},
$$

\footnotetext{
1 In the following we shall no longer stress the dependence of the inverse placement map upon the considered current time and therefore use $\hat{\chi}_{\alpha}^{-1}$ instead of $\chi_{\alpha}^{-1}(\cdot, t)$
} 


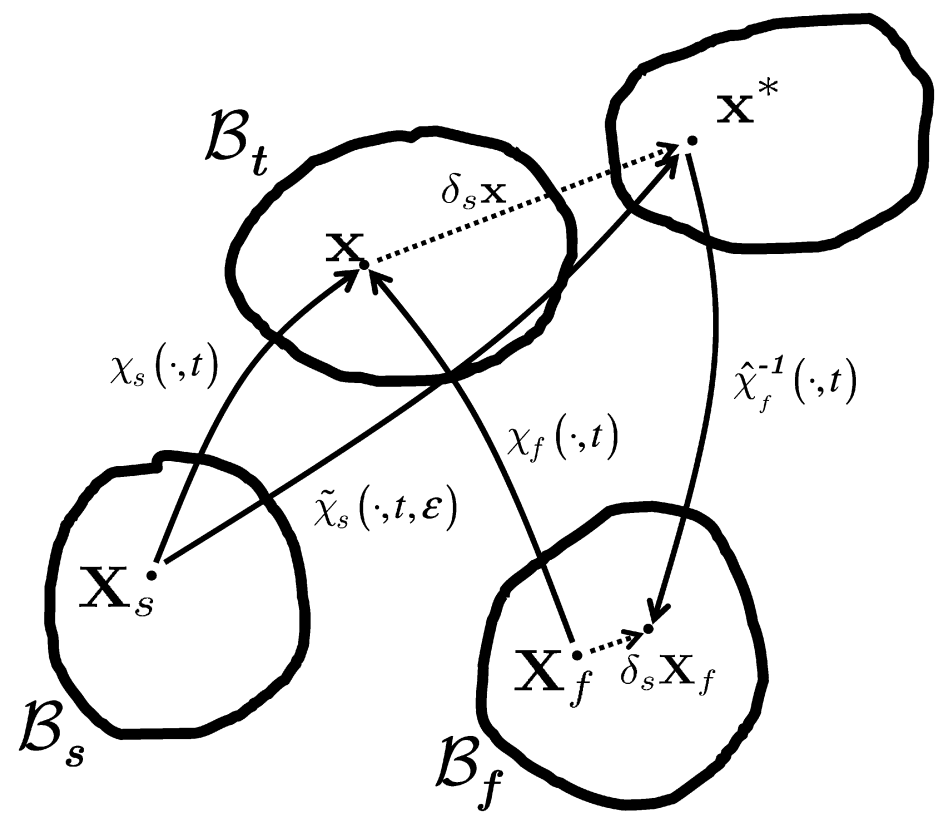

Fig. 1. Reference configurations of solid and fluid components are shown with the actual configurations of the mixture: before and after a solid variation. Curved arrows represent placement or inverse placement functions. Dotted arrows represent solid variations. Let us remark that in a solid-variation the places $\mathbf{x}$ and $\mathbf{x}^{*}$ are the images of the same solid particle $\mathbf{X}_{s}$ and of different fluid particle, i.e., $\mathbf{X}_{f}=\hat{\chi}_{f}^{-1}\left(\tilde{\chi}_{s}\left(\mathbf{X}_{s}, t, 0\right), t\right)$ and $\mathbf{X}_{f}=\hat{\chi}_{f}^{-1}\left(\tilde{\chi}_{s}\left(\mathbf{X}_{s}, t, \varepsilon\right), t\right)$. In the figure it is shown the scheme of a solid-variation as it is prescribed by the definition (2.2). It is evident that an analogous scheme for the fluid variation can be achieved simply interchanging the symbols $s$ and $f$ of this figure.

as the partial time derivative of the fields in (1.4), respectively. This means that $d_{\alpha} / d t$ is the time derivative keeping fixed the particle $\mathbf{X}_{\alpha}$ and $\partial / \partial t$ is the time derivative when the variable $\mathbf{x}$ holds constant. In the same way it is possible to treat the derivatives with respect to the space variables. We define

$$
\nabla_{\mathbf{X}_{s}}, \quad \nabla_{\mathbf{X}_{f}}, \quad \nabla_{\mathbf{x}},
$$

as the gradient operators of the fields in (1.4), respectively. This means that $\nabla_{\mathbf{X}_{\alpha}}$ is the gradient operator with respect to the vector $\mathbf{X}_{\alpha}$ and $\nabla_{\mathbf{x}}$ is the gradient operator with respect to the vector $\mathbf{x}$. Classical examples of the introduced operators end the present subsection.

The inverse functions introduced in (1.2) define an identity vector function $\mathbf{I}_{d}$ over the reference configuration of the $\alpha$-th constituent, viz.,

$$
\mathbf{X}_{\alpha}=\mathbf{I}_{d}\left(\mathbf{X}_{\alpha}\right)=\hat{\chi}_{\alpha}^{-1}(\mathbf{x}, t)=\hat{\chi}_{\alpha}^{-1}\left(\chi_{\alpha}\left(\mathbf{X}_{\alpha}, t\right), t\right),
$$

that is important in order to derive the following relation with the use of chain derivative rule, i.e.,

$$
\frac{d_{\alpha}}{d t} \mathbf{I}_{d}=\mathbf{0}=\left(\nabla_{\mathbf{x}} \hat{\chi}_{\alpha}^{-1}\right) \frac{d_{\alpha}}{d t} \chi_{\alpha}+\frac{\partial \hat{\chi}_{\alpha}^{-1}}{\partial t} .
$$

The derivative with respect to time of (1.1), following the motion of the $\alpha$-species, gives the velocity fields $\mathbf{v}_{\alpha}$ of the same species and the derivatives with respect to the particles $\mathbf{X}_{\alpha}$ of (1.1) or (1.3) give the deformation gradients $\mathbf{F}_{\alpha}$, i.e.,

$$
\mathbf{v}_{s}=\frac{d_{s} \chi_{s}}{d t}, \quad \mathbf{v}_{f}=\frac{d_{f} \chi_{f}}{d t}, \quad \mathbf{F}_{s}=\nabla_{\mathbf{X}_{s}} \chi_{s}=\mathbf{I}+\mathbf{H}_{s}, \quad \mathbf{F}_{f}=\nabla_{\mathbf{X}_{f}} \chi_{f}=\mathbf{I}+\mathbf{H}_{f},
$$

where $\mathbf{H}_{s}$ and $\mathbf{H}_{f}$ are the displacement gradients of the solid and of the fluid, respectively. In the rest of the paper the symmetric part $\mathbf{E}_{s}$,

$$
\mathbf{E}_{s}=\frac{1}{2}\left(\mathbf{H}_{s}+\mathbf{H}_{s}^{T}\right)
$$


of the displacement gradient $\mathbf{H}_{s}$ of the solid constituent will be extensively used. Besides, the derivative with respect to time of the deformation gradient $\mathbf{F}_{\alpha}$ and of the determinant $J_{\alpha}=\operatorname{det} \mathbf{F}_{\alpha}$ of it, i.e. the Jacobians of the transformations defined in (1.1), following the motion of the species $\alpha$ are,

$$
\begin{aligned}
& \frac{d_{\alpha}}{d t} \mathbf{F}_{\alpha}=\frac{d_{\alpha}}{d t} \nabla_{\mathbf{x}_{\alpha}} \chi_{\alpha}=\nabla_{\mathbf{x}_{\alpha}} \frac{d_{\alpha}}{d t} \chi_{\alpha}=\nabla_{\mathbf{x}_{\alpha}} \mathbf{v}_{\alpha}=\left(\nabla_{\mathbf{x}} \mathbf{v}_{\alpha}\right) \mathbf{F}_{\alpha}, \\
& \frac{d_{\alpha}}{d t} J_{\alpha}=\frac{d_{\alpha}}{d t} \operatorname{det} \mathbf{F}_{\alpha}=J_{\alpha} \operatorname{tr}\left(\nabla_{\mathbf{x}} \mathbf{v}_{\alpha}\right)=J_{\alpha} \nabla_{\mathbf{x}} \cdot \mathbf{v}_{\alpha},
\end{aligned}
$$

where $\operatorname{tr}$ and $\nabla_{\mathbf{x}}$. are the trace and the divergence operators, respectively; Eq. (1.10) comes from the derivative chain rule and Eq. (1.11) from a well known algebra formula derived for a general matrix $A=\hat{A}(t)$,

$$
\frac{\partial}{\partial t} \operatorname{det} \hat{A}=\operatorname{tr}\left[\frac{\partial \hat{A}}{\partial t} \hat{A}^{-1}\right] \operatorname{det} \hat{A} \text {. }
$$

\subsection{Mass balances}

In a solid-fluid mixture the total mass of each species, i.e. $M_{\alpha}$, are constant whether we follow the motion of the related constituent because no transfer of mass is permitted between one species and the other. This means that Eq. (1.11) yields,

$$
\frac{d_{\alpha}}{d t} M_{\alpha}=\frac{d_{\alpha}}{d t} \int_{\mathcal{B}_{t}} \varrho_{\alpha}=\frac{d_{\alpha}}{d t} \int_{\mathcal{B}_{\alpha}} \varrho_{\alpha}^{*}=\int_{\mathcal{B}_{\alpha}} \frac{d_{\alpha}}{d t}\left(\varrho_{\alpha} J_{\alpha}\right)=\int_{\mathcal{B}_{t}} \frac{d_{\alpha} \varrho_{\alpha}}{d t}+\varrho_{\alpha} \nabla_{\mathbf{x}} \cdot \mathbf{v}_{\alpha}=0,
$$

where $\varrho_{\alpha}$ is the partial mass density of the species $\alpha$, or the mass of the species $\alpha$ per unit volume of the mixture in the actual configuration, and $\varrho_{\alpha}^{*}=\varrho_{\alpha} J_{\alpha}$ is the referential partial mass density of the species $\alpha$, or the mass of the species $\alpha$ per unit volume of the mixture in the reference configurations of species $\alpha$. A localisation of (1.13) gives rise to the mass balance equations of each species,

$$
\frac{d_{\alpha} \varrho_{\alpha}}{d t}+\varrho_{\alpha} \nabla_{\mathbf{x}} \cdot\left(\mathbf{v}_{\alpha}\right)=\frac{\partial \varrho_{\alpha}}{\partial t}+\nabla_{\mathbf{x}} \cdot\left(\varrho_{\alpha} \mathbf{v}_{\alpha}\right)=0
$$

The sum over $\alpha$ of the equations given in (1.14) yields to the total mass conservation law, i.e.,

$$
\frac{\partial \varrho}{\partial t}+\nabla_{\mathbf{x}} \cdot(\varrho \mathbf{v})=0
$$

where,

$$
\varrho=\varrho_{s}+\varrho_{f}, \quad \xi_{s}=\frac{\varrho_{s}}{\varrho}, \quad \xi_{f}=\frac{\varrho_{f}}{\varrho}, \quad \xi_{s}+\xi_{f}=1, \quad \mathbf{v}=\xi_{s} \mathbf{v}_{s}+\xi_{f} \mathbf{v}_{f},
$$

$\xi_{\alpha}$ is the so-called mass fraction of the species $\alpha$ and $\mathbf{v}$ is the so-called barycentric velocity of the mixture.

\section{A variational approach for the derivation of balance equations}

In order to derive momentum balance equations, the action $\mathcal{A}$ should be defined in terms of the density (i.e., per unit volume) $\mathcal{L}$ of a Lagrangian $L$ as follows,

$$
\mathcal{A}=\int_{t_{0}}^{t_{1}} L=\int_{t_{0}}^{t_{1}} \int_{\mathcal{B}_{t}} \mathcal{L}, \quad \mathcal{L}=\frac{1}{2} \varrho_{s}\left|\mathbf{v}_{s}\right|^{2}+\frac{1}{2} \varrho_{f}\left|\mathbf{v}_{f}\right|^{2}-\varrho \Psi ;
$$

$\mathcal{L}$ is, as usual, the difference between the densities of the kinetic and of the internal energy, and $\Psi$ is the internal energy per unit mass of the mixture.

For a monophasic material the variation of the action functional plus the virtual work of the external forces is imposed to be zero and the balance equations are, as a consequence, derived according with a kind of localisation procedure. In this case the varied place is defined by

$$
\mathbf{x}^{*}=\tilde{\chi}(\mathbf{X}, t, \varepsilon)=\chi(\mathbf{X}, t)+\varepsilon \eta(\mathbf{X}, t),
$$


where the vector field $\eta$ is arbitrary subjected to the conditions $\eta\left(\mathbf{X}, t_{0}\right)=\eta\left(\mathbf{X}, t_{1}\right)=0$. We note that such variations are intended to be computed for a fixed particle $\mathbf{X}$. We also note that $\varepsilon$ has the role to parameterise such variation.

In a mixture theory the generalisation of this approach can bring almost two ambiguities. The first one regards the placement map $\left(\chi_{s}\right.$ or $\chi_{f}$ ) which has to be varied, the second is about the particle one should follow in the variation. Among other approaches, we use that belonging to Gouin (1990), Gavrilyuk et al. (1998), Gouin and Gavrilyuk (1999), Gavrilyuk and Gouin (1999), Gouin and Ruggeri (2003) and to Gavrilyuk (2005). Apparently, specifying a variation scheme affects the form of the derived field equations. In this approach, two families of variations are used. In the first family we consider the variation of only one placement field, e.g. $\chi_{\alpha}$, and fix the particle $\mathbf{X}_{\alpha}$, i.e.,

$$
\left\{\begin{array}{l}
\mathbf{x}=\tilde{\chi}_{\alpha}\left(\mathbf{X}_{\alpha}, t, \varepsilon\right)=\chi_{\alpha}\left(\mathbf{X}_{\alpha}, t\right)+\varepsilon \eta_{\alpha}\left(\mathbf{X}_{\alpha}, t\right) \\
\mathbf{x}=\chi_{\beta}\left(\mathbf{X}_{\beta}, t\right)=\chi_{\beta}\left(\hat{\chi}_{\beta}^{-1}(\mathbf{x}, t), t\right)=\chi_{\beta}\left(\hat{\chi}_{\beta}^{-1}\left(\tilde{\chi}_{\alpha}\left(\mathbf{X}_{\alpha}, t, \varepsilon\right), t\right), t\right) .
\end{array}\right.
$$

As for the case of monophasic materials the variation is parameterised by $\varepsilon$ and Eqs. (1.1) and (1.2) have been used. We call $\delta_{\alpha}$ the $\alpha$-th variation operator as it is given by (2.2), i.e.,

$$
\delta_{\alpha} A(\varepsilon)=A(\varepsilon)-A(0) \Longrightarrow \delta_{\alpha} \mathbf{x}=\delta_{\alpha} \tilde{\chi}_{\alpha}=\delta_{\alpha} \chi_{\beta}=\varepsilon \eta_{\alpha}\left(\mathbf{X}_{\alpha}, t\right),
$$

where $A$ is a general function of the parameter $\varepsilon$. The second family of variations is defined simply changing the roles of $s$ and $f$. In Fig. 1 we show the scheme of a solid variation; even in this case the fluid variation can be derived changing the role of $s$ and $f$. We prove that the $\alpha$-th variation of the $\alpha$-th fields are,

$$
\begin{aligned}
& \delta_{\alpha} \mathbf{v}_{\alpha}=\delta_{\alpha} \frac{d_{\alpha} \tilde{\chi}_{\alpha}}{d t}=\varepsilon \frac{d_{\alpha} \eta_{\alpha}}{d t}, \\
& \delta_{\alpha} \mathbf{F}_{\alpha}=\delta_{\alpha} \nabla_{\mathbf{x}_{\alpha}} \tilde{\chi}_{\alpha}=\varepsilon \nabla_{\mathbf{x}_{\alpha}} \eta_{\alpha}, \\
& \delta_{\alpha} J_{\alpha}=\delta_{\alpha} \operatorname{det} \mathbf{F}_{\alpha}=\varepsilon \operatorname{tr}\left[\left(\nabla_{\mathbf{x}_{\alpha}} \eta_{\alpha}\right) \mathbf{F}_{\alpha}^{-1}\right] J_{\alpha}=\varepsilon \operatorname{tr}\left[\nabla_{\mathbf{x}} \eta_{\alpha}\right] J_{\alpha}=\varepsilon J_{\alpha} \nabla_{\mathbf{x}} \cdot \eta_{\alpha}, \\
& \delta_{\alpha} \varrho_{\alpha}=-\frac{\varrho_{\alpha}^{*}}{J_{\alpha}^{2}} \delta_{\alpha} J_{\alpha}=-\varepsilon \frac{\varrho_{\alpha}^{*}}{J_{\alpha}^{2}} J_{\alpha} \nabla_{\mathbf{x}} \cdot \eta_{\alpha}=-\varepsilon \varrho_{\alpha} \nabla_{\mathbf{x}} \cdot \eta_{\alpha},
\end{aligned}
$$

and the $\alpha$-variation of the $\beta$-fields are,

$$
\begin{aligned}
\delta_{\alpha} \mathbf{v}_{\beta} & =\varepsilon\left(\nabla_{\mathbf{x}} \hat{\mathbf{v}}_{\beta}\right) \eta_{\alpha}, \\
\delta_{\alpha} \mathbf{F}_{\beta} & =\varepsilon\left(\nabla_{\mathbf{x}} \hat{\mathbf{F}}_{\beta}\right) \eta_{\alpha}, \\
\delta_{\alpha} J_{\beta} & =\varepsilon\left(\nabla_{\mathbf{x}} \hat{J}_{\beta}\right) \cdot \eta_{\alpha}, \\
\delta_{\alpha} \varrho_{\beta} & =\varepsilon\left(\nabla_{\mathbf{x}} \hat{\varrho}_{\beta}\right) \cdot \eta_{\alpha} .
\end{aligned}
$$

The field equations of a solid-fluid mixture will be derived by computing the two kind of variations $\delta_{s}$ and $\delta_{f}$ of the action $\mathcal{A}$,

$$
\delta_{\alpha} \mathcal{A}=\delta_{\alpha} \int_{t_{0}}^{t_{1}} \int_{\mathcal{B}_{t}} \mathcal{L}=\delta_{\alpha} \int_{t_{0}}^{t_{1}} \int_{\mathcal{B}_{\alpha}} J_{\alpha} \mathcal{L}=\int_{t_{0}}^{t_{1}} \int_{\mathcal{B}_{\alpha}} \delta_{\alpha}\left(J_{\alpha} \mathcal{L}\right)=\int_{t_{0}}^{t_{1}} \int_{\mathcal{B}_{\alpha}} \varepsilon J_{\alpha}\left(\nabla_{\mathbf{x}} \cdot \eta_{\alpha}\right) \mathcal{L}+J_{\alpha} \delta_{\alpha} \mathcal{L},
$$

where a change of variables was necessary to pass the variation operator into the integral. The $\alpha$-variation of the Lagrangian density can be computed as follows,

$$
\delta_{\alpha} \mathcal{L}=\frac{1}{2}\left(\delta_{\alpha} \varrho_{s}\right)\left|\mathbf{v}_{s}\right|^{2}+\varrho_{s} \mathbf{v}_{s} \cdot\left(\delta_{\alpha} \mathbf{v}_{s}\right)+\frac{1}{2}\left(\delta_{\alpha} \varrho_{f}\right)\left|\mathbf{v}_{f}\right|^{2}+\varrho_{f} \mathbf{v}_{f} \cdot\left(\delta_{\alpha} \mathbf{v}_{f}\right)-\left(\delta_{\alpha} \varrho\right) \Psi-\varrho\left(\delta_{\alpha} \Psi\right) .
$$

The internal energy per unit mass is assumed to be a function of the partial mass density $\varrho_{f}$ of the fluid and of the deformation gradient $\mathbf{F}_{s}$ of the solid, that holds

$$
\Psi=\Psi\left(\varrho_{f}, \mathbf{F}_{s}\right) ; \quad \delta_{\alpha} \Psi=\frac{\partial \Psi}{\partial \varrho_{f}} \delta_{\alpha} \varrho_{f}+\frac{\partial \Psi}{\partial \mathbf{F}_{s}}: \delta_{\alpha} \mathbf{F}_{s} .
$$

Such a dependency brakes the formal symmetry between the solid and the fluid constituent and obliges us to split the derivation of the consequences of the two variations, that are,

$$
\delta_{\alpha} \mathcal{A}=\varepsilon \int_{t_{0}}^{t_{1}} \int_{\mathcal{B}_{t}}\left[-\varrho_{\alpha} \frac{d_{\alpha}}{d t} \mathbf{v}_{\alpha}+\nabla_{\mathbf{x}} \cdot \mathbf{T}_{\alpha}+\mathbf{m}_{\alpha}\right] \cdot \eta_{\alpha}+\varepsilon \int_{t_{0}}^{t_{1}} \int_{\mathcal{B}_{t}}\left\{\left[\left(\frac{1}{2} \varrho_{\beta}\left|\mathbf{v}_{\beta}\right|^{2}-\varrho_{\beta} \Psi\right) \mathbf{I}-\mathbf{T}_{\alpha}\right] \mathbf{n}\right\} \cdot \eta_{\alpha},
$$


where,

$$
\left\{\begin{array}{l}
\mathbf{m}_{s}=\mathbf{m}=\varrho_{f} \frac{\partial \Psi}{\partial \mathbf{F}_{s}}:\left(\nabla_{\mathbf{x}} \mathbf{F}_{s}\right)-\varrho_{s} \frac{\partial \Psi}{\partial \varrho_{f}}\left(\nabla_{\mathbf{x}} \varrho_{f}\right), \quad \mathbf{m}_{f}=-\mathbf{m}, \\
\mathbf{T}_{s}=\varrho \frac{\partial \Psi}{\partial \mathbf{F}_{s}} \mathbf{F}_{s}^{T}, \quad \mathbf{T}_{f}=-\varrho \varrho_{f} \frac{\partial \Psi}{\partial \varrho_{f}} \mathbf{I}=-p_{f} \mathbf{I},
\end{array}\right.
$$

where $\mathbf{m}_{\alpha}$ (or $\mathbf{m}$ ) and $\mathbf{T}_{\alpha}$ are the bulk interaction forces and the Cauchy stress tensors of the species $\alpha$. The derivation of (2.3)-(2.4) is given explicitly in Appendix A. We now assume that the $\alpha$-th variation of the action plus the virtual work that external forces spend on such variations, including the Darcy dissipative term, is imposed to be zero, i.e.,

$$
\delta_{\alpha} \mathcal{A}-\varepsilon \int_{t_{0}}^{t_{1}} \int_{\mathcal{B}_{t}} D\left[\mathbf{v}_{\alpha}-\mathbf{v}_{\beta}\right] \cdot \eta_{\alpha}+\varepsilon \int_{t_{0}}^{t_{1}} \int_{\partial \mathcal{B}_{t}} \mathbf{t}_{\alpha}^{\mathrm{ext}} \cdot \eta_{\alpha}=0
$$

where $\mathbf{t}_{\alpha}^{\text {ext }}$ is the external force acting on the border of the mixture and $D$ is the permeability coefficient. We remark that the integral over the boundary which appears in (2.5) can be written for the fluid constituent in the following form,

$$
\int_{\partial \mathcal{B}_{t}}\left\{\left[\left(\frac{1}{2} \varrho_{s}\left|\mathbf{v}_{s}\right|^{2}-\varrho_{s} \Psi\right) \mathbf{I}-\mathbf{T}_{f}\right] \mathbf{n}+\mathbf{t}_{f}^{\mathrm{ext}}\right\} \cdot \eta_{f}=\int_{\partial \mathcal{B}_{t}}\left\{\left[\left(\mathcal{L}-\frac{\partial \mathcal{L}}{\partial \varrho_{f}} \varrho_{f}\right) \mathbf{I}\right] \mathbf{n}-\mathbf{t}_{f}^{\mathrm{ext}}\right\} \cdot \eta_{f} .
$$

An analogous form holds true also for the solid where the dependence of $\mathcal{L}$ on $\mathbf{F}_{s}$ should be accounted for. Requiring these terms to be vanishing implies the so-called trasversality conditions, which naturally arise when the boundary of the domain is not fixed in the variation (see e.g. Courant and Hilbert, 1953). The variations $\eta_{s}$ and $\eta_{f}$ are assumed to be arbitrary so that the following equations hold,

$$
\left\{\begin{array}{l}
\varrho_{s} \frac{d_{s}}{d t} \mathbf{v}_{s}=\nabla_{\mathbf{x}} \cdot \mathbf{T}_{s}+\mathbf{m}+D\left(\mathbf{v}_{f}-\mathbf{v}_{s}\right) \\
\varrho_{f} \frac{d_{f}}{d t} \mathbf{v}_{f}=\nabla_{\mathbf{x}} \cdot \mathbf{T}_{f}-\mathbf{m}-D\left(\mathbf{v}_{f}-\mathbf{v}_{s}\right),
\end{array}\right.
$$

which are also known as the linear momentum balance equations of the mixture.

\section{The constitutive equations with pre-stress}

\subsection{The internal energy}

In this section we will provide constitutive equations for the Cauchy stress tensors $\mathbf{T}_{\alpha}$ of both constituents and for the interaction force $\mathbf{m}$. In (2.4), we derived the relations between them and the specific internal energy $\Psi$. However, an explicit characterisation of $\Psi$ is missed. Firstly, we decompose the partial mass densities $\varrho_{\alpha}$ into $\varrho_{0}^{\alpha}$ and $\tilde{\varrho}_{\alpha}$, i.e. the partial mass density in the unperturbed configuration and the partial mass density due to the perturbation, respectively,

$$
\varrho_{\alpha}=\varrho_{0}^{\alpha}+\tilde{\varrho}_{\alpha},
$$

and consider only the perturbation $\tilde{\varrho}_{\alpha}$ in the functional dependence of $\Psi, \varrho_{0}^{\alpha}$ being known from the beginning. Secondly, we require the specific internal energy to be an objective function. It is well known in continuum mechanics (see, e.g., Liu, 2002) that an objective scalar function can depend on a tensor field according with a very specific rule, for instance,

$$
\Psi=\bar{\Psi}\left(\mathbf{G}_{s}, \tilde{\varrho}_{f}\right), \quad \mathbf{G}_{s}=\frac{1}{2}\left(\mathbf{F}_{s}^{T} \mathbf{F}_{s}-\mathbf{I}\right)=\frac{1}{2}\left(\mathbf{H}_{s}+\mathbf{H}_{s}^{T}+\mathbf{H}_{s}^{T} \mathbf{H}_{s}\right)=\mathbf{E}_{s}+\frac{1}{2} \mathbf{H}_{s}^{T} \mathbf{H}_{s},
$$

where $\mathbf{G}_{s}$ is the solid Green-Saint-Venant strain tensor that is an objective tensor in the sense that its matrix representation is the same for all the reference frames. Thus, a possible functional dependence of $\Psi$ can be the following polynomial decomposition,

$$
\Psi=\Psi_{0}+\boldsymbol{\Psi}_{s}: \mathbf{G}_{s}+\Psi_{f} \tilde{\varrho}_{f}+\left(\boldsymbol{\Psi}_{s s}: \mathbf{G}_{s}\right): \mathbf{G}_{s}+\Psi_{f f} \tilde{\varrho}_{f}^{2}+\tilde{\varrho}_{f} \Psi_{s f}: \mathbf{G}_{s}+\cdots,
$$

where $\Psi_{0}, \Psi_{f}$ and $\Psi_{f f}$ are scalar parameters, $\boldsymbol{\Psi}_{s}$ and $\boldsymbol{\Psi}_{s f}$ are general 2 nd order tensor parameters and $\boldsymbol{\Psi}_{s s}$ is a general 4th order tensor parameter. The dots at the end of (3.2) indicate that, in principle, we could consider any further terms 
of this expansion. However, we will see that such a truncation is sufficient to keep all the terms that remain after the linearity assumption, that is the same to say that the perturbation is sufficiently small.

The second assumption is, in fact, about the perturbation. We assume that the displacements $\mathbf{u}_{\alpha}$ and the displacement gradients $\mathbf{H}_{\alpha}$ are sufficiently small to neglect quadratic terms in the balance equations (2.7). In order to take into account such an approximation we decompose not only the deformation gradient (see Eqs. (1.8)) but also the mass densities (see Eq. (3.1)) into two parts, the first related to the unperturbed fields with vanishing $\mathbf{u}_{\alpha}$ and $\mathbf{H}_{\alpha}$ and the second related to the perturbation. The advantage of this representation stays in the fact that we have just a linear combination of the perturbed fields and the quadratic terms can at most arise in the form of the specific internal energy:

$$
J_{\alpha}=1+\operatorname{tr} \mathbf{H}_{\alpha}, \quad \varrho_{\alpha}=\frac{\varrho_{0}^{\alpha}}{J_{\alpha}}=\varrho_{0}^{\alpha}-\varrho_{0}^{\alpha} \operatorname{tr} \mathbf{H}_{\alpha}, \quad \tilde{\varrho}_{\alpha}=-\varrho_{0}^{\alpha} \operatorname{tr} \mathbf{H}_{\alpha}, \quad \xi_{0}^{\alpha}=\frac{\varrho_{0}^{\alpha}}{\varrho_{0}},
$$

where $\xi_{0}^{\alpha}$ is the mass fraction of the species $\alpha$ in the unperturbed configuration. The function $\bar{\Psi}$ is assumed quadratic in the perturbed fields and is represented as follows,

$$
\Psi=\Psi_{0}+\boldsymbol{\Psi}_{s}: \mathbf{E}_{s}+\frac{1}{2} \Psi_{s}:\left(\mathbf{H}_{s}^{T} \mathbf{H}_{s}\right)+\Psi_{f} \tilde{\varrho}_{f}+\left(\boldsymbol{\Psi}_{s s}: \mathbf{E}_{s}\right): \mathbf{E}_{s}+\Psi_{f f} \tilde{\varrho}_{f}^{2}+\tilde{\varrho}_{f} \boldsymbol{\Psi}_{s f}: \mathbf{E}_{s},
$$

\subsection{The constitutive equations}

We are now interested to give an interpretation of the coefficients of Eq. (3.4) in order to be able to measure them. To do this, we can evaluate the form of the Cauchy stress tensors and of the interaction force via the (2.4), i.e.,

$$
\begin{aligned}
& \mathbf{T}_{s}=\varrho_{0} \boldsymbol{\Psi}_{s}+\varrho_{0} \tilde{\varrho}_{f} \boldsymbol{\Psi}_{s f}+\varrho_{0}\left(\mathbf{H}_{s} \boldsymbol{\Psi}_{s}+\boldsymbol{\Psi}_{s} \mathbf{H}_{s}^{T}\right)+2 \varrho_{0} \boldsymbol{\Psi}_{s s}: \mathbf{E}_{s}+\tilde{\varrho}_{f} \boldsymbol{\Psi}_{s}-\varrho_{0}^{s}\left(\operatorname{tr} \mathbf{E}_{s}\right) \boldsymbol{\Psi}_{s}, \\
& \mathbf{T}_{f}=-\mathbf{I} \varrho\left[\Psi_{f}+2 \Psi_{f f} \tilde{\varrho}_{f}+\boldsymbol{\Psi}_{s f}: \mathbf{E}_{s}\right] \varrho_{0}^{f},
\end{aligned}
$$

where the 4th order tensor $\boldsymbol{\Psi}_{s s}$ is now constrained to fulfil certain symmetries that can be easily represented with the indicial notation as follows,

$$
\left(\boldsymbol{\Psi}_{s s}\right)_{i j h k}=\left(\boldsymbol{\Psi}_{s s}\right)_{j i h k}=\left(\boldsymbol{\Psi}_{s s}\right)_{h k i j}
$$

The derivation of (3.5) is deferred in the appendix. The representation (3.5) and (3.6) of the Cauchy stress tensors allow us to interpret $\boldsymbol{\Psi}_{s}, \Psi_{f}, \boldsymbol{\Psi}_{s s}$ and $\Psi_{f f}$ as follows. If we do not have any perturbation, then the states of the Cauchy stress tensors are the pre-stress states, i.e.,

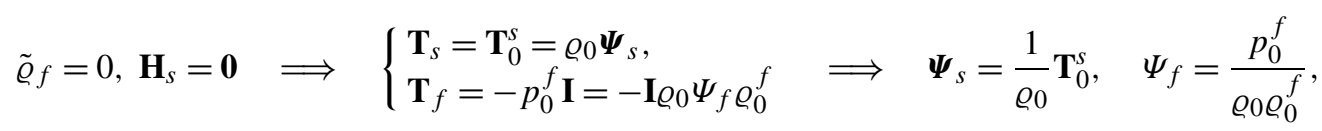

where $p_{0}^{f}$ is the pressure of the fluid species before the deformation, i.e. the pre-stress of the fluid and $\mathbf{T}_{0}^{s}$ is the Cauchy stress tensor of the solid species before the deformation, i.e. the pre-stress of the solid. If the solid is absent, then the mixture is made from the pure fluid and if the pre-stress of the fluid is null, then the classical constitutive equation of a perfect fluid is assumed to hold, i.e.,

$$
p_{0}^{f}=0, \text { no solid } \Longrightarrow \mathbf{T}_{f}=-\kappa_{f} \tilde{\varrho}_{f} \mathbf{I}=-2 \varrho_{0}^{f} \varrho_{0}^{f} \Psi_{f f} \tilde{\varrho}_{f} \mathbf{I} \quad \Longrightarrow \quad \Psi_{f f}=\frac{\kappa_{f}}{2\left(\varrho_{0}^{f}\right)^{2}},
$$

where $\kappa_{f}$ is the compressibility coefficient of such fluid. If the fluid is absent, then the mixture is made from the pure solid and if the pre-stress of the solid is null, then the classical constitutive equation of a linear and elastic solid is assumed to hold, i.e.,

$$
\mathbf{T}_{0}^{s}=\mathbf{0} \text {, no fluid } \Longrightarrow \mathbf{T}_{s}=\mathbb{C}_{s}: \mathbf{E}_{s}=2 \varrho_{0}^{s} \Psi_{s s}: \mathbf{E}_{s} \quad \Longrightarrow \quad \boldsymbol{\Psi}_{s s}=\frac{1}{2 \varrho_{0}^{s}} \mathbb{C}_{s},
$$

where $\mathbb{C}_{s}$ is the 4 th order elasticity tensor of such solid. We remark again that the numerical values of $\kappa_{f}$ and $\mathbb{C}_{s}$ are related to the given pre-stressed reference configuration of the mixture and it is therefore not possible to use values measured in other configurations. 
With such an interpretation we have,

$$
\Psi=\Psi_{0}+\frac{1}{\varrho_{0}} \mathbf{T}_{0}^{s}: \mathbf{E}_{s}+\frac{p_{0}^{f}}{\varrho_{0} \varrho_{0}^{f}} \tilde{\varrho}_{f}+\left(\frac{1}{2 \varrho_{0}^{s}} \mathbb{C}_{s}: \mathbf{E}_{s}\right): \mathbf{E}_{s}+\frac{1}{2} \frac{1}{\varrho_{0}} \mathbf{T}_{0}^{s}:\left(\mathbf{H}_{s}^{T} \mathbf{H}_{s}\right)+\frac{\kappa_{f}}{2\left(\varrho_{0}^{f}\right)^{2}} \tilde{\varrho}_{f}^{2}+\tilde{\varrho}_{f} \boldsymbol{\Psi}_{s f}: \mathbf{E}_{s},
$$

and, as a consequence, the (2.4) yields,

$$
\begin{aligned}
& \mathbf{T}_{s}=\mathbf{T}_{0}^{s}+\tilde{\varrho}_{f}\left[\varrho_{0} \boldsymbol{\Psi}_{s f}+\frac{1}{\varrho_{0}} \mathbf{T}_{0}^{s}\right]+\mathbf{H}_{s} \mathbf{T}_{0}^{s}+\mathbf{T}_{0}^{s} \mathbf{H}_{s}^{T}-\left(\operatorname{tr} \mathbf{E}_{s}\right) \frac{\varrho_{0}^{s}}{\varrho_{0}} \mathbf{T}_{0}^{s}+\frac{1}{\xi_{0}^{s}} \mathbb{C}_{s}: \mathbf{E}_{s}, \\
& \mathbf{T}_{f}=-\mathbf{I}\left[p_{0}^{f}+\left(\frac{p_{0}^{f}}{\varrho_{0}^{f}}+\frac{p_{0}^{f}}{\varrho_{0}}+\frac{\kappa_{f}}{\xi_{0}^{f}}\right) \tilde{\varrho}_{f}+\frac{p_{0}^{f}}{\varrho_{0}} \tilde{\varrho}_{s}+\varrho_{0} \varrho_{0}^{f} \boldsymbol{\Psi}_{s f}: \mathbf{E}_{s}\right], \\
& \mathbf{m}=p_{0}^{f} \frac{\xi_{0}^{s}}{\varrho_{0}^{f}}\left(\nabla_{\mathbf{x}} \tilde{\varrho}_{f}\right)-\xi_{0}^{f} \mathbf{T}_{0}^{s}: \nabla_{\mathbf{x}} \mathbf{H}_{s} .
\end{aligned}
$$

The constitutive equations of the mixture are completely characterised once one assigns the constitutive parameters $D$, $\boldsymbol{\Psi}_{s f}, \kappa_{f}$ and $\mathbb{C}_{s}$. We remark that such parameters are referred to the given reference configuration and therefore are related to the prescribed state of pre-stress. It would be very important to know the relation between the constitutive parameters $D, \boldsymbol{\Psi}_{s f}, \kappa_{f}$ and $\mathbb{C}_{s}$ and the pre-stresses $\mathbf{T}_{0}^{s}$ and $p_{0}^{f}$. However, not always it is possible to derive a general relation between them. Such relations are often an unknown of the problem and the measures must be done independently.

The scalar $D$ and the tensor $\boldsymbol{\Psi}_{s f}$ represent the dissipative and the non-dissipative interaction between the species of the mixture. $\boldsymbol{\Psi}_{s f}$ is symmetric and we need 6 constitutive parameters to characterise it. If the only spherical part of $\mathbf{H}^{s}$ is relevant, then only one parameter is needed to model this kind of interaction and we could postulate,

$$
\boldsymbol{\Psi}_{s f}=-\varrho_{0}^{s} \gamma_{s f} \mathbf{I}
$$

where $\gamma_{s f}$ is this unique constitutive parameter. In this paper, even though the non-spherical part of $\mathbf{H}_{s}$ is considered, we claim the validity of (3.12). Furthermore, the fourth order elasticity tensor $\mathbb{C}_{s}$ is assumed to be isotropic in such a way $\mathbb{C}_{S}$ can be written in the following form,

$$
\mathbb{C}_{s} \mathbf{E}_{s}=2 \mu \mathbf{E}_{s}+\lambda \operatorname{tr}\left(\mathbf{E}_{s}\right) \mathbf{I},
$$

where $\lambda$ and $\mu$ are the Lamé coefficients evaluated for the given solid component and for the given state of pre-stress.

The two isotropic constitutive assumptions (3.12) and (3.13) imply also a restriction on the states of pre-stress. In fact, we recall that $\boldsymbol{\Psi}_{s f}$ and $\mathbb{C}_{s}$ are related not only to the kind of materials considered to build up the mixture but also to the state of pre-stress and therefore it is reasonable to assume,

$$
\mathbf{T}_{0}^{s}=-p_{0}^{s} \mathbf{I}, \quad \mathbf{T}_{0}^{f}=-p_{0}^{f} \mathbf{I} .
$$

If we insert the (3.12), (3.13) and (3.14) into (3.9), (3.10) and (3.11), then we obtain an explicit form of the constitutive equations as follows,

$$
\begin{aligned}
& \mathbf{T}^{s}=-p_{0}^{s} \mathbf{I}+2\left(\mu \frac{1}{\xi_{0}^{s}}-p_{0}^{s}\right) \mathbf{E}_{s}+\left(\lambda \frac{1}{\xi_{0}^{s}}+\xi_{0}^{s} p_{0}^{s}\right)\left(\operatorname{tr} \mathbf{E}_{s}\right) \mathbf{I}-\left(\varrho_{0}^{s} \varrho_{0} \gamma_{s f}+\frac{p_{0}^{s}}{\varrho_{0}}\right) \tilde{\varrho}_{f} \mathbf{I}, \\
& \mathbf{T}^{f}=-p_{0}^{f} \mathbf{I}-\left(\frac{\kappa_{f}}{\xi_{0}^{f}}+\frac{p_{0}^{f}}{\varrho_{0}^{f}}+\frac{p_{0}^{f}}{\varrho_{0}}\right) \tilde{\varrho}_{f} \mathbf{I}+\left(\varrho_{0}^{f} \varrho_{0} \gamma_{s f}+\frac{p_{0}^{f}}{\varrho_{0}}\right)\left(\varrho_{0}^{s} \operatorname{tr} \mathbf{E}_{s}\right) \mathbf{I}, \\
& \mathbf{m}=p_{0}^{f} \frac{\xi_{0}^{s}}{\varrho_{0}^{f}} \nabla \tilde{\varrho_{f}}+p_{0}^{s} \frac{\xi_{0}^{f}}{\varrho_{0}^{s}} \nabla\left(\varrho_{0}^{s} \operatorname{tr} \mathbf{E}_{s}\right)+D\left(\mathbf{v}^{s}-\mathbf{v}^{f}\right),
\end{aligned}
$$

where we group the dissipative Darcy term into the functional dependence of the interaction force $\mathbf{m}$. The set of constitutive equations expressed in (3.15) is the result of the assumptions stated in the text. We remark that in the model of Wilmanski $(2000 ; 1996)$ the interaction force $\mathbf{m}$ is represented solely by the Darcy term even though the presence of the pre-stress is considered. The reason is that Wilmanski adopts the approximation of simple mixture and the constitutive quantities cannot depend upon the gradient of densities. A further difference is that, in the model 
of Wilmanski, the pre-stress is constrained to be small and it is neglected when it multiplies the linear quantities $\mathbf{E}_{s}$ or $\tilde{\varrho}_{f}$. In this approach we can relax the condition of the smallness of pre-stresses and such terms take a non-trivial role. Besides, the form of the conservative part of the specific energy (3.8) guarantees that the states of stress of solid and fluid reduce to those prescribed in (3.14) when no deformation occurs.

A final remark concerns the implication of the smallness assumption of the perturbed fields. Once we regard the velocity fields $\mathbf{v}_{\alpha}$ to be sufficiently small to be considered linear in the field equations, then the mass balances (1.14) becomes,

$$
\frac{\partial \tilde{\varrho}_{\alpha}}{\partial t}+\varrho_{0}^{\alpha} \nabla_{\mathbf{x}} \cdot\left(\mathbf{v}_{\alpha}\right)+\tilde{\varrho}_{\alpha} \nabla_{\mathbf{x}} \cdot\left(\mathbf{v}_{\alpha}\right)+\left(\nabla_{\mathbf{x}} \tilde{\varrho}_{\alpha}\right) \cdot \mathbf{v}_{\alpha} \simeq \frac{\partial \tilde{\varrho}_{\alpha}}{\partial t}+\varrho_{0}^{\alpha} \nabla_{\mathbf{x}} \cdot\left(\mathbf{v}_{\alpha}\right),
$$

and the inertial terms into the momenta balance take the following form, i.e.,

$$
\varrho_{\alpha} \frac{d_{\alpha}}{d t} \mathbf{v}_{\alpha}=\varrho_{0}^{\alpha}\left[\frac{\partial}{\partial t} \mathbf{v}_{\alpha}+\left(\nabla_{\mathbf{x}} \mathbf{v}_{\alpha}\right) \mathbf{v}_{\alpha}\right]+\tilde{\varrho}_{\alpha} \frac{d_{\alpha}}{d t} \mathbf{v}_{\alpha} \simeq \varrho_{0}^{\alpha} \frac{\partial}{\partial t} \mathbf{v}_{\alpha} .
$$

\subsection{The linearised field equations}

If we insert the constitutive equations (3.15) into the balance equations (2.7) and (3.16) with the use of (3.17), the linearised field equations take the forms,

$$
\begin{aligned}
\frac{\partial \tilde{\varrho}_{s}}{\partial t}= & -\varrho_{0}^{s} \nabla \cdot \mathbf{v}_{s}, \\
\frac{\partial \tilde{\varrho}_{f}}{\partial t}= & -\varrho_{0}^{f} \nabla \cdot \mathbf{v}_{f}, \\
\varrho_{0}^{s} \frac{\partial \mathbf{v}_{s}}{\partial t}= & 2\left(\mu \frac{1}{\xi_{0}^{s}}-p_{0}^{s}\right) \nabla \cdot \mathbf{E}_{s}+\left(\lambda \frac{1}{\xi_{0}^{s}}+\xi_{0}^{s} p_{0}^{s}-\xi_{0}^{f} p_{0}^{s}\right) \nabla\left(\operatorname{tr} \mathbf{E}_{s}\right) \\
& -\left(\varrho_{0}^{s} \varrho_{0}^{f} \varrho_{0} \gamma_{s f}+p_{0}^{s} \xi_{0}^{f}+p_{0}^{f} \xi_{0}^{s}\right) \frac{1}{\varrho_{0}^{f}} \nabla \tilde{\varrho}_{f}-D\left(\mathbf{v}_{s}-\mathbf{v}_{f}\right), \\
\varrho_{0}^{f} \frac{\partial \mathbf{v}_{f}}{\partial t}= & -\left(\frac{\kappa f}{\xi_{0}^{f}}+2 \frac{p_{0}^{f}}{\varrho_{0}}\right) \nabla \tilde{\varrho}_{f}+\left(\varrho_{0}^{s} \varrho_{0}^{f} \varrho_{0} \gamma_{s f}+p_{0}^{s} \xi_{0}^{f}+p_{0}^{f} \xi_{0}^{s}\right) \nabla\left(\operatorname{tr} \mathbf{E}_{s}\right)+D\left(\mathbf{v}_{s}-\mathbf{v}_{f}\right),
\end{aligned}
$$

By derivation of (3.20) and (3.21) with respect to time and using the continuity equations (3.18) and (3.19) we derive,

$$
\left\{\begin{array}{l}
\varrho_{0}^{s} \frac{\partial^{2} \mathbf{v}_{s}}{\partial t^{2}}=\left(\mu \frac{1}{\xi_{0}^{s}}-p_{0}^{s}\right) \nabla \cdot \nabla \mathbf{v}_{s}+\left[\varrho_{0}^{s} \tilde{\gamma}_{s s}+p_{0}^{s}-\mu \frac{1}{\xi_{0}^{s}}\right] \nabla\left(\nabla \cdot \mathbf{v}_{s}\right)+\sqrt{\varrho_{0}^{s} \varrho_{0}^{f}} \tilde{\gamma}_{s f} \nabla\left(\nabla \cdot \mathbf{v}_{f}\right)-D \frac{\partial}{\partial t}\left(\mathbf{v}_{s}-\mathbf{v}_{f}\right), \\
\varrho_{0}^{f} \frac{\partial^{2} \mathbf{v}_{f}}{\partial t^{2}}=\varrho_{0}^{f} \tilde{\gamma}_{f f} \nabla\left(\nabla \cdot \mathbf{v}_{f}\right)+\tilde{\gamma}_{s f} \sqrt{\varrho_{0}^{s} \varrho_{0}^{f}} \nabla\left(\nabla \cdot \mathbf{v}_{s}\right)+D \frac{\partial}{\partial t}\left(\mathbf{v}_{s}-\mathbf{v}_{f}\right),
\end{array}\right.
$$

where

$$
\tilde{\gamma}_{s s}=\frac{\lambda \frac{1}{\xi_{0}^{s}}+2 \mu \frac{1}{\xi_{0}^{s}}}{\varrho_{0}^{s}}-\frac{p_{0}^{s}}{\varrho_{0}^{s}}\left(1+2 \xi_{0}^{f}\right), \quad \tilde{\gamma}_{s f}=\sqrt{\varrho_{0}^{s} \varrho_{0}^{f}}\left[\varrho_{0} \gamma_{s f}+\frac{p_{0}^{s}}{\varrho_{0}^{s} \varrho_{0}}+\frac{p_{0}^{f}}{\varrho_{0}^{f} \varrho_{0}}\right], \quad \tilde{\gamma}_{f f}=\frac{\kappa_{f}}{\xi_{0}^{f}}+2 \frac{p_{0}^{f}}{\varrho_{0}} .
$$

Eqs. (3.22) constitute the field equations for the proposed isothermal problem.

\section{Propagation of monochromatic plane waves}

In this section we use the linearised field Eqs. (3.22) to study the propagation of monochromatic plane waves in an infinite porous medium, considering the separate contribution of transverse, $S$-waves, and longitudinal waves, $P 1$ and $P 2$-waves, respectively (see Biot, 1956a, 1956b). Starting from this analysis, stability of equilibrium, when considering transverse or longitudinal wave perturbations, is discussed.

Plane wave propagation in porous materials is particularly relevant when considering how they allow for identifying the constitutive characteristics of stratified layers. However this is not the only interesting question at which plane wave propagation analysis may address in geomechanics. Management and abandonment of highly-pressurised leached-out 
salt caverns is also strongly related to this topic, when considering, for instance, the behaviour of these structures in the occurrence of the earthquake phenomenon. Both during their life cycle and in abandonment (see e.g. Cosenza et al., 1999), seismic perturbations of equilibrium can induce local dilatancy or local compaction of the so called DisturbedRock-Zone (DRZ, see e.g. Cosenza et al., 1999), in the vicinity of the cavern walls. The first one enhances diffusion of the stored fluid (typically hydrocarbons or brine) through the porous medium, the second one local compaction and consequently over-pressurisation of the cavern itself. Both these occurrences determine high risk environmental conditions, since they favourite stored hydrocarbons to escape outside of the cavern, possibly causing subsidence phenomena of the overburden, or they may trigger fracture propagation in the salt formation, which could raise up to the external surface.

\subsection{A general dispersion relation}

We are interested in a solution of the form,

$$
\tilde{\varrho}_{\alpha}=\hat{\varrho}_{\alpha} e^{i(\omega t-\mathbf{k} \cdot \mathbf{x})}, \quad \mathbf{v}_{\alpha}=\hat{\mathbf{v}}_{\alpha} e^{i(\omega t-\mathbf{k} \cdot \mathbf{x})}, \quad \alpha=s, f,
$$

where $\omega$ is the frequency and $\mathbf{k}$ the wave vector, both possibly complex, $\hat{\varrho}_{\alpha}$ and $\hat{\mathbf{v}}_{\alpha}$ are constant amplitudes of the monochromatic plane waves.

Substituting the above expressions into Eqs. (3.22), we obtain,

$$
\left\{\begin{array}{l}
-\varrho_{0}^{s} \omega^{2} \hat{\mathbf{v}}_{s}=-\left(\mu \frac{1}{\xi_{0}^{s}}-p_{0}^{s}\right) k^{2} \hat{\mathbf{v}}_{s}-\left[\varrho_{0}^{s} \tilde{\gamma}_{s s}+p_{0}^{s}-\mu \frac{1}{\xi_{0}^{s}}\right]\left(\hat{\mathbf{v}}_{s} \cdot \mathbf{k}\right) \mathbf{k}-\sqrt{\varrho_{0}^{s} \varrho_{0}^{f}} \tilde{\gamma}_{s f}\left(\hat{\mathbf{v}}_{f} \cdot \mathbf{k}\right) \mathbf{k}-i \omega D\left(\hat{\mathbf{v}}^{s}-\hat{\mathbf{v}}^{f}\right), \\
-\varrho_{0}^{f} \omega^{2} \hat{\mathbf{v}}_{f}=-\varrho_{0}^{f} \tilde{\gamma}_{f f}\left(\hat{\mathbf{v}}_{f} \cdot \mathbf{k}\right) \mathbf{k}-\sqrt{\varrho_{0}^{s} \varrho_{0}^{f}} \tilde{\gamma}_{s f}\left(\hat{\mathbf{v}}_{s} \cdot \mathbf{k}\right) \mathbf{k}+i \omega D\left(\hat{\mathbf{v}}^{s}-\hat{\mathbf{v}}^{f}\right),
\end{array}\right.
$$

and, arranging the terms in a different form:

$$
\left(\begin{array}{ll}
\mathbf{A}_{s s} & \mathbf{A}_{s f} \\
\mathbf{A}_{s f} & \mathbf{A}_{f f}
\end{array}\right)\left(\begin{array}{c}
\hat{\mathbf{v}}_{s} \\
\hat{\mathbf{v}}_{f}
\end{array}\right)=\left(\begin{array}{l}
\mathbf{0} \\
\mathbf{0}
\end{array}\right)
$$

where,

$$
\begin{aligned}
& \mathbf{A}_{s s}=\left(\left(\mu \frac{1}{\xi_{0}^{s}}-p_{0}^{s}\right) k^{2}+i \omega D-\varrho_{0}^{s} \omega^{2}\right) \mathbf{I}+\left(\varrho_{0}^{s} \tilde{\gamma}_{s s}+p_{0}^{s}-\mu \frac{1}{\xi_{0}^{s}}\right)(\mathbf{k} \otimes \mathbf{k}), \\
& \mathbf{A}_{s f}=-i \omega D \mathbf{I}+\sqrt{\varrho_{0}^{s} \varrho_{0}^{f}} \tilde{\gamma}_{s f}(\mathbf{k} \otimes \mathbf{k}), \\
& \mathbf{A}_{f f}=\left(i \omega D-\varrho_{0}^{f} \omega^{2}\right) \mathbf{I}+\varrho_{0}^{f} \tilde{\gamma}_{f f}(\mathbf{k} \otimes \mathbf{k}),
\end{aligned}
$$

and $\mathbf{I}$ is the three dimensional identity tensor. The (4.2) form a system of six scalar equations which admits non-trivial solutions if and only if,

$$
\operatorname{Det}\left(\begin{array}{cc}
\mathbf{A}_{s s} & \mathbf{A}_{s f} \\
\mathbf{A}_{s f} & \mathbf{A}_{f f}
\end{array}\right)=0
$$

Eq. (4.3) identifies the dispersion relation; a suitable simplification can be obtained decomposing the amplitudes of the velocity fields $\hat{\mathbf{v}}_{\alpha}$ into a solenoidal $\hat{\mathbf{v}}_{\alpha}^{\perp}$ and an irrotational $\hat{v}_{\alpha}^{\|} \mathbf{k}$ part, i.e.,

$$
\hat{\mathbf{v}}_{\alpha}=\hat{\mathbf{v}}_{\alpha}^{\perp}+\hat{v}_{\alpha}^{\|} \mathbf{k}, \quad \mathbf{k} \cdot \hat{\mathbf{v}}_{\alpha}^{\perp}=0, \quad \nabla \cdot\left(\hat{\mathbf{v}}_{\alpha}^{\perp} e^{i(\omega t-\mathbf{k} \cdot \mathbf{x})}\right)=0, \quad \nabla \times\left(\hat{v}_{\alpha}^{\|} \mathbf{k} e^{i(\omega t-\mathbf{k} \cdot \mathbf{x})}\right)=0,
$$

corresponding to the transversal and longitudinal velocity fields, respectively. We will analyse these two cases in the next two subsections.

\subsection{Transverse waves}

\subsubsection{The dispersion relation}

The transverse part of the velocity amplitude fields $\hat{\mathbf{v}}_{s}^{\perp}$ and $\hat{\mathbf{v}}_{f}^{\perp}$, from (4.3) and (4.4), satisfies the following system of equations,

$$
\left.\begin{array}{l}
-\omega^{2} \varrho_{0}^{s} \hat{\mathbf{v}}_{s}^{\perp}+\left(\mu \frac{1}{\xi_{0}^{s}}-p_{0}^{s}\right) k^{2} \hat{\mathbf{v}}_{s}^{\perp}+i \omega D\left(\hat{\mathbf{v}}_{s}^{\perp}-\hat{\mathbf{v}}_{f}^{\perp}\right)=0 \\
-\omega^{2} \varrho_{0}^{f} \hat{\mathbf{v}}_{f}^{\perp}-i \omega D\left(\hat{\mathbf{v}}_{s}^{\perp}-\hat{\mathbf{v}}_{f}^{\perp}\right)=0
\end{array}\right\}
$$


where $k$ is the modulus of the wave vector $\mathbf{k}$. A non-trivial solution to the previous equations exists provided that,

$$
\omega^{4} \varrho_{0}^{s} \varrho_{0}^{f}-i \omega^{3} \varrho_{0} D-\omega^{2} k^{2} \varrho_{0}^{f}\left(\mu \frac{1}{\xi_{0}^{s}}-p_{0}^{s}\right)+i \omega k^{2} D\left(\mu \frac{1}{\xi_{0}^{s}}-p_{0}^{s}\right)=0,
$$

that is the dispersion relation for transverse waves.

\subsubsection{The zero and infinite limits}

It is instructive to examine the character of Eq. (4.6) in the zero and infinite limits.

In the zero limit,

$$
\omega \rightarrow 0, \quad k=|\mathbf{k}| \rightarrow 0, \quad \omega / k \text { finite, }
$$

the phase velocity $V_{p h}=|\operatorname{Re}(\omega / k)|$ is given by,

$$
V_{p h}\left(\mu-p_{0}^{s}-V_{p h}^{2} \varrho_{0}\right)=0, \quad V_{p h 0}=0, \quad V_{p h 1, p h 2}= \pm \sqrt{\frac{\mu \frac{1}{\xi_{0}^{s}}-p_{0}^{s}}{\varrho_{0}}} .
$$

The velocity $V_{p h 0}=0$ corresponds to the static solution, ${ }^{2}$ while the non-vanishing phase velocities exist provided that,

$$
p_{0}^{s}<\mu \frac{1}{\xi_{0}^{s}} .
$$

In this case, which corresponds to consider the limit $D \rightarrow \infty$, the relative motion between the two constituents vanishes, see Eqs. (4.5).

In the infinite limit,

$$
\omega \rightarrow \infty, \quad k=|\mathbf{k}| \rightarrow \infty, \quad \omega / k \text { finite }
$$

the phase velocity is given by,

$$
V_{p h}^{2}\left(\mu \frac{1}{\xi_{0}^{s}}-p_{0}^{s}-V_{p h}^{2} \varrho_{0}^{s}\right)=0, \quad V_{p h 0}=0, \quad V_{p h 1, p h 2}= \pm \sqrt{\frac{\mu \frac{1}{\xi_{0}^{s}}-p_{0}^{s}}{\varrho_{0}^{s}}} .
$$

Even in this case the velocity $V_{p h 0}=0$ corresponds to the static solution, while the non-zero phase velocities exist under the same restriction as Eq. (4.9). In this case, which also corresponds to consider $D \rightarrow 0$, there is no effect of the coupling between the solid and the fluid components, see Eqs. (4.5).

\subsubsection{Asymptotic of the roots}

In the general case, the exact solution of Eq. (4.6) can, of course, be obtained; however it is of interest to discuss the behaviour of the solutions in the case of real and small $k$. Real values of $k$ correspond to pure oscillatory initial data. Let us now expand $\omega(k)$ in powers of $k$ around $k=0$,

$$
\omega(k)=\omega_{0}+\omega_{1} k+\omega_{2} k^{2}+\cdots ;
$$

substituting this expansion into Eq. (4.6) and equating terms of the same power (de Socio et al., 2003), the expression of the four roots are obtained,

$$
\omega^{(1)}=0, \quad \omega^{(2,3)}= \pm \sqrt{\frac{\mu \frac{1}{\xi_{0}^{s}}-p_{0}^{s}}{\varrho_{0}}} k+\mathrm{o}(k), \quad \omega^{(4)}=i\left(v-\left(\mu \frac{1}{\xi_{0}^{s}}-p_{0}^{s}\right) \frac{\xi_{0}^{f}}{\nu \varrho_{0}^{s}} k^{2}\right)+\mathrm{o}\left(k^{2}\right),
$$

where

$$
v=D \frac{\varrho_{0}}{\varrho_{0}^{s} \varrho_{0}^{f}} .
$$

The leading terms of the first three roots give the solutions discussed in the zero limit case; $\omega^{(2,3)}$ correspond to the sound modes, the duality arising from the two ways of propagation. In the last root the frequency is purely

\footnotetext{
2 In the literature such condition is also called of marginal stability, see e.g. Ref. (Fu and Ogden, 1999).
} 
imaginary up to the second order. This means that the disturbances do not propagate. The normal mode solutions can be obtained by using the previous roots in Eqs. (4.5) and solving for the fluctuation amplitudes $\hat{\mathbf{v}}_{s}^{\perp}, \hat{\mathbf{v}}_{f}^{\perp}$. For each mode the dispersion law is an expression for $\omega(k)$.

Real values of $\omega$ correspond to a disturbance maintained by a source of fixed frequency (bvp). The analytical solution for $k$,

$$
k^{2}=\frac{\omega \varrho_{0}^{s}}{\mu \frac{1}{\xi_{0}^{s}}-p_{0}^{s}} \frac{\omega-i \nu}{\omega-i \xi_{0}^{s} \nu},
$$

can be expanded considering small $\omega$ as,

$$
k(\omega)=k_{0}+k_{1} \omega+k_{2} \omega^{2}+\cdots .
$$

The two roots are given by,

$$
k^{(1,2)}= \pm \sqrt{\frac{\varrho_{0}}{\mu \frac{1}{\xi_{0}^{s}}-p_{0}^{s}}} \omega\left(1 \mp i \frac{\varrho_{0}^{f}}{2 v \varrho_{0}^{s}} \omega\right)+\mathrm{o}\left(\omega^{2}\right) .
$$

This mode has the characteristics of a propagating mode, that can be carried out only in a bounded domain.

\subsection{Longitudinal waves}

\subsubsection{The dispersion relation}

The equations for the longitudinal velocity amplitudes are,

$$
\left\{\begin{array}{l}
{\left[i \omega D-\omega^{2} \varrho_{0}^{s}+\varrho_{0}^{s} \tilde{\gamma}_{s s} k^{2}\right] \hat{v}_{s}^{\|}+\left[\sqrt{\varrho_{0}^{s} \varrho_{0}^{f}} \tilde{\gamma}_{s f} k^{2}-i \omega D\right] \hat{v}_{f}^{\|}=0,} \\
{\left[\sqrt{\varrho_{0}^{s} \varrho_{0}^{f}} \tilde{\gamma}_{s f} k^{2}-i \omega D\right] \hat{v}_{s}^{\|}+\left[i \omega D-\omega^{2} \varrho_{0}^{f}+\varrho_{0}^{f} \tilde{\gamma}_{f f} k^{2}\right] \hat{v}_{f}^{\|}=0 .}
\end{array}\right.
$$

A non-trivial solution of the previous two coupled equations exists if and only if,

$$
\omega^{4}-i \omega^{3} v-\omega^{2} k^{2}\left(C_{+}+C_{-}\right)+i v \omega k^{2} C_{0}+k^{4} C_{+} C_{-}=0,
$$

where,

$$
\begin{aligned}
& C_{0}=\xi_{0}^{s} \tilde{\gamma}_{s s}+\xi_{0}^{f} \tilde{\gamma}_{f f}+2 \sqrt{\xi_{0}^{s} \xi_{0}^{f}} \tilde{\gamma}_{s f}, \\
& C_{+}+C_{-}=\tilde{\gamma}_{s s}+\tilde{\gamma}_{f f}, \\
& C_{+} C_{-}=\tilde{\gamma}_{s s} \tilde{\gamma}_{f f}-\tilde{\gamma}_{s f}^{2} ;
\end{aligned}
$$

Eqs. (4.16) and (4.17) imply,

$$
C_{ \pm}=\frac{\tilde{\gamma}_{s s}+\tilde{\gamma}_{f f} \pm \sqrt{\left(\tilde{\gamma}_{s s}-\tilde{\gamma}_{f f}\right)^{2}+4 \tilde{\gamma}_{s f}^{2}}}{2} .
$$

Eq. (4.14) can now be written as follows,

$$
-i v \frac{\omega}{k^{2}}\left(\frac{\omega^{2}}{k^{2}}-C_{0}\right)+\left[\left(\frac{\omega^{2}}{k^{2}}-C_{+}\right)\left(\frac{\omega^{2}}{k^{2}}-C_{-}\right)\right]=0 .
$$

\subsubsection{The zero and infinite limits}

In the zero limit defined in (4.7), Eq. (4.18) can be solved in terms of phase velocity,

$$
V_{p h 0}=0, \quad V_{p h 1, p h 2}= \pm \sqrt{C_{0}},
$$

that corresponds to the situation in which the two components of the mixture move together $(D \rightarrow \infty)$.

On the other hand, in the infinite limit defined in (4.10), Eq. (4.18) is solved by,

$$
V_{p h 1, p h 2, p h 3, p h 4}= \pm \sqrt{C_{ \pm}},
$$

and we remark again that this limit corresponds to the classical result for non-dispersive case $(D \rightarrow 0)$. 


\subsubsection{Asymptotic of the roots}

In analogy with the previous results, for real $k$, we look at the solution of Eq. (4.18) in the same form of (4.12). The expressions of the four roots are, in this case,

$$
\omega^{(1,2)}= \pm \sqrt{C_{0}} k+\mathrm{o}(k), \quad \omega^{(3)}=\mathrm{o}(k), \quad \omega^{(4)}=i v-i\left[\frac{C_{0}-\left(C_{+}+C_{-}\right)}{v}\right] k^{2}+\mathrm{o}\left(k^{2}\right) .
$$

The first pair of roots correspond to the sound mode, the duality, as before, arising from the two ways of propagation. The leading term of the last root is pure imaginary: this means that the disturbance relaxes in time toward the equilibrium state (see Section 4.3.4).

For real $\omega$ the (4.14) is bi-quadratic, an analytical solution is not a difficult task and corresponds to a disturbance maintained by a source of fixed frequency. For real and small $\omega$, we put the expansion,

$$
k(\omega)=k_{0}+\omega^{1 / 2} k_{1}+\omega k_{2} \cdots
$$

into the dispersion relation (4.18) and find,

$$
k_{0}=0, \quad k_{1}= \pm \sqrt{\frac{v C_{0}}{2 C_{+} C_{-}}}(1-i), \quad k_{2}=\hat{k}_{2}\left(C_{ \pm}, C_{0}, v\right),
$$

where $\hat{k}_{2}$ is a certain function easy to determine, but complicated to write down.

\subsubsection{Remark}

Let us introduce the following matrix,

$$
\mathbf{B}=\left(\begin{array}{ll}
\tilde{\gamma}_{s s} & \tilde{\gamma}_{s f} \\
\tilde{\gamma}_{s f} & \tilde{\gamma}_{f f}
\end{array}\right)
$$

We want to show that the existence of a non-vanishing phase velocity in the zero and infinite limits, analysed in this subsection, is equivalent to the positive definiteness of the matrix $\mathbf{B}$. This is an easy task. In fact, if the matrix $\mathbf{B}$ is definite positive than we have

$$
\tilde{\gamma}_{s s}>0, \quad \tilde{\gamma}_{f f}>0, \quad \tilde{\gamma}_{s s} \tilde{\gamma}_{f f}-\tilde{\gamma}_{s f}^{2}>0 .
$$

Thus, (4.15) is a positive quadratic form in the quantities $\sqrt{\xi_{0}^{s}}$ and $\sqrt{\xi_{0}^{f}}$ that, together with (4.16) and (4.17), gives,

$$
C_{0}>0, \quad C_{+}+C_{-}>0, \quad C_{+} C_{-}>0, \quad C_{+}+C_{-}-C_{0}>0
$$

these last imply the positiveness of $C_{0}, C_{+}$and $C_{-}$.

We will see that the conditions (4.25) are the same as those derived from the linear stability analysis in the next section.

\section{Linear stability analysis}

\subsection{The general case}

In this subsection we discuss the linear stability of the equilibrium state, with respect to plane wave perturbation. Let now consider the following change of variable in the dispersion equations (4.6) and (4.14),

$$
\omega \rightarrow-i \omega,
$$

these read now as follows,

$$
\begin{aligned}
& \omega\left[\omega^{3}+v \omega^{2}+k^{2} \frac{\mu \frac{1}{\xi_{0}^{s}}-p_{0}^{s}}{\varrho_{0}^{s}} \omega+k^{2} \frac{\mu \frac{1}{\xi_{0}^{s}}-p_{0}^{s}}{\varrho_{0}} v\right]=0, \\
& \omega^{4}+v \omega^{3}+\omega^{2} k^{2}\left(C_{+}+C_{-}\right)+\omega \nu C_{0} k^{2}+k^{4} C_{+} C_{-}=0 .
\end{aligned}
$$

If the real part $\omega_{r}$ of the frequency $\omega=\omega_{r}+i \omega_{i}$ is negative, then the perturbations in the form of plane waves are attenuated in time. Note that this does not mean that the system is stable under all kind of perturbations since we 
are limiting our attention to plane waves. On the other hand positive values of $\omega_{r}$ imply the system to be unstable. Besides, if $\omega_{r}=0$ the system is called neutrally (or marginally) stable.

For transverse waves the negativeness of the real part of the frequency is assured from the Routh-Hurwitz theorem (see, e.g., Ref. (Gantmacher, 1960)) by the following conditions,

$$
D>0, \quad p_{0}^{s}<\mu \frac{1}{\xi_{0}^{s}} .
$$

The first condition can be interpreted as a consequence of the Second Law of Thermodynamics and simply tells us that the dissipation due to the Darcy term in the constitutive equation $(3.15)_{3}$ is not able to produce energy. Notice that the second restriction is the same one as that deduced in Eq. (4.9).

For longitudinal waves the Routh-Hurwitz theorem assures the negativeness of the real part of frequency according to the following conditions,

$$
\text { (1) } D>0, \quad \text { (2) } C_{+}+C_{-}-C_{0}>0, \quad \text { (3) } C_{0}\left(C_{+}+C_{-}-C_{0}\right)-C_{+} C_{-}>0, \quad \text { (4) } C_{-} C_{+}>0 \text {. }
$$

Condition (1) is the same as (5.4) 1 ; from the definitions (4.15)-(4.17) and (3.23), it is easy to show that conditions (2) and (4) can be represented as follows,

$$
\begin{aligned}
& 3 p_{0}^{s}<\lambda \frac{1}{\xi_{0}^{s}}+2 \mu \frac{1}{\xi_{0}^{s}}+\varrho_{0} \varrho_{0}^{s} \varrho_{0}^{s}\left(\frac{\kappa_{f}}{\varrho_{0}^{f} \varrho_{0}^{f}}-2 \gamma_{s f}\right), \\
& {\left[\lambda \frac{1}{\xi_{0}^{s}}+2 \mu \frac{1}{\xi_{0}^{s}}-p_{0}^{s}\left(1+2 \xi_{0}^{f}\right)\right]\left(\varrho_{0} \kappa_{f}+2 \xi_{0}^{f} p_{0}^{f}\right)>\left(\varrho_{0}^{s} \varrho_{0}^{f} \varrho_{0} \gamma_{s f}+\xi_{0}^{f} p_{0}^{s}+\xi_{0}^{s} p_{0}^{f}\right)^{2},}
\end{aligned}
$$

whilst condition (3) is always satisfied. We remark that conditions (5.4) 2 , (5.6) and (5.7) are explicit or implicit restrictions on the values of the prestresses $p_{0}^{s}$ and $p_{0}^{f}$. Whether $p_{0}^{s}=p_{0}^{f}=0$, the following condition must be satisfied, i.e.,

$$
\gamma_{s f}<\frac{\sqrt{\kappa_{f}(\lambda+2 \mu)}}{\varrho_{0}^{s} \varrho_{0}^{f} \sqrt{\varrho_{0}^{s}}} .
$$

Inequality (5.8) tells us that, in order to guarantee the stability of the mixture, the interaction between the species, namely the parameter $\gamma_{s f}$, cannot be too strong. We remark that such condition can be guaranteed by the positive definiteness of the strain energy $\Psi$ as it is shown in the next section.

\section{Positive definiteness of the internal energy}

We discussed the stability of an equilibrium state induced by the prestress of a solid-fluid mixture for infinitesimal disturbances in the form of a general plane wave. In this section, we analyse stability conditions with respect to infinitesimal homogeneous perturbations in the absence of initial stresses. This can be achieved assuming the positive definiteness of the strain energy. To do this we study the Hessian matrix of $\Psi$ with respect to the non-dimensional variables $\mathbf{G}_{s}$ and $x_{f}=\tilde{\varrho}_{f} / \varrho_{0}^{f}$, i.e.,

$$
\begin{aligned}
& \frac{\partial^{2} \Psi}{\partial \mathbf{G}_{s} \partial \mathbf{G}_{s}}=2 \boldsymbol{\Psi}_{s s}=\frac{1}{\varrho_{0}^{s}} \mathbb{C}_{s}, \quad \frac{\partial^{2} \Psi}{\partial G_{s}^{i j} \partial G_{s}^{h k}}=\frac{1}{\varrho_{0}^{s}}\left[\mu\left(\delta_{i k} \delta_{j h}+\delta_{i h} \delta_{j k}\right)+\lambda \delta_{i j} \delta_{h k}\right], \\
& \frac{\partial^{2} \Psi}{\partial \mathbf{G}_{s} \partial x_{f}}=\boldsymbol{\Psi}_{s f} \varrho_{0}^{f}=-\varrho_{0}^{s} \varrho_{0}^{f} \gamma_{s f} \mathbf{I}, \quad \frac{\partial^{2} \Psi}{\partial G_{s}^{i j} \partial x_{f}}=-\varrho_{0}^{s} \varrho_{0}^{f} \gamma_{s f} \delta_{i j}, \\
& \frac{\partial^{2} \Psi}{\partial x_{f}^{2}}=2 \bar{\Psi}_{f f} \varrho_{0}^{f} \varrho_{0}^{f}=\kappa_{f},
\end{aligned}
$$

where the indicial notation are now useful in order to calculate the eigenvalues of the Hessian matrix, i.e., 


$$
\frac{\partial \Psi}{\partial \mathbf{x} \partial \mathbf{x}}=\left(\begin{array}{ccccccc}
\frac{2 \mu+\lambda}{\varrho_{0}^{s}} & 0 & 0 & \frac{\lambda}{\varrho_{0}^{s}} & 0 & \frac{\lambda}{\varrho_{0}^{s}} & -\varrho_{0}^{s} \varrho_{0}^{f} \gamma_{s f} \\
0 & \frac{\mu}{\varrho_{0}^{s}} & 0 & 0 & 0 & 0 & 0 \\
0 & 0 & \frac{\mu}{\varrho_{0}^{s}} & 0 & 0 & 0 & 0 \\
\frac{\lambda}{\varrho_{0}^{s}} & 0 & 0 & \frac{2 \mu+\lambda}{\varrho_{0}^{s}} & 0 & \frac{\lambda}{\varrho_{0}^{s}} & -\varrho_{0}^{s} \varrho_{0}^{f} \gamma_{s f} \\
0 & 0 & 0 & 0 & \frac{\mu}{\varrho_{0}^{s}} & 0 & 0 \\
\frac{\lambda}{\varrho_{0}^{s}} & 0 & 0 & \frac{\lambda}{\varrho_{0}^{s}} & 0 & \frac{2 \mu+\lambda}{\varrho_{0}^{s}} & -\varrho_{0}^{s} \varrho_{0}^{f} \gamma_{s f} \\
-\varrho_{0}^{s} \varrho_{0}^{f} \gamma_{s f} & 0 & 0 & -\varrho_{0}^{s} \varrho_{0}^{f} \gamma_{s f} & 0 & -\varrho_{0}^{s} \varrho_{0}^{f} \gamma_{s f} & \kappa_{f}
\end{array}\right) ;
$$

the 7-dimensional variable $\mathbf{x}$ is defined as follows,

$$
\mathbf{x}=\left\{G_{s}^{11}, G_{s}^{12}, G_{s}^{13}, G_{s}^{22}, G_{s}^{23}, G_{s}^{33}, x_{f}\right\} .
$$

The eigenvalues of the Hessian matrix are,

$$
\begin{aligned}
& \alpha_{1,2,3}=\frac{\mu}{\varrho_{0}^{s}}, \quad \alpha_{4,5}=2 \frac{\mu}{\varrho_{0}^{s}}, \\
& \alpha_{6,7}=\frac{1}{2}\left[\left(\frac{2 \mu+3 \lambda}{\varrho_{0}^{s}}+\kappa_{f}\right) \pm \sqrt{\left(\frac{2 \mu+3 \lambda}{\varrho_{0}^{s}}+\kappa_{f}\right)^{2}+4\left(3\left(\varrho_{0}^{s} \varrho_{0}^{f} \gamma_{s f}\right)^{2}-\kappa_{f}\left(\frac{2 \mu+3 \lambda}{\varrho_{0}^{s}}\right)\right)}\right]
\end{aligned}
$$

that are all positive if and only if,

$$
\mu>0, \quad \frac{2 \mu+3 \lambda}{\varrho_{0}^{s}}+\kappa_{f}>0, \quad \gamma_{s f}<\frac{1}{\varrho_{0}^{s} \varrho_{0}^{f}} \sqrt{\frac{\kappa_{f}(2 \mu+3 \lambda)}{3 \varrho_{0}^{s}}},
$$

that guarantee the conditions (5.8).

\section{Numerical results}

\subsection{A numerical example}

In Fig. 2, we represent (5.4) 2 , (5.6) and (5.7) in terms of a graphic in which the coordinate axes are the prestresses $p_{0}^{s}$ and $p_{0}^{f}$. If constitutive parameters do not depend from the pre-stress, ${ }^{3}$ then conditions $(5.4)_{2}$ and (5.6) are halfplanes and condition (5.7) defines a region bounded by an hyperbola. If we use the numerical example of Garg et al. (1974), then the conservative interaction parameter $\gamma_{s f}$ is deduced,

$$
\gamma_{s f}=4.37 \mathrm{~m}^{8} / \mathrm{Kg}^{2} \mathrm{~s}^{2} .
$$

However, such parameter is not measured in experiments and it is parameterised in the next subsection. In order to build an effective numerical example, we consider a porous and saturated sandstones (see Ref. (Wilmanski, 2004)) in the following numerical setting, ${ }^{4}$

$$
\left\{\begin{array}{l}
\varrho_{0}^{s}=2100 \mathrm{Kg} / \mathrm{m}^{3}, \quad \varrho_{0}^{f}=230 \mathrm{Kg} / \mathrm{m}^{3}, \quad D=2.6 \times 10^{7} \mathrm{Kg} / \mathrm{m}^{3} \mathrm{~s}, \quad n=0.23, \\
\gamma_{s f}=0 \mathrm{~m}^{8} / \mathrm{Kg}^{2} \mathrm{~s}^{2}, \quad \lambda=2 \mathrm{GPa}, \quad \mu=9 \mathrm{GPa}, \quad \kappa_{f}=8 \times 10^{4} \mathrm{~m}^{2} / \mathrm{s}^{2},
\end{array}\right.
$$

where $n$ is the porosity, also called volume fraction of the fluid part, and $\gamma_{s f}$ is assumed to vanish. The graphic is shown in Fig. 2, where the intersection of the regions defined in $(5.4)_{2},(5.6)$, and (5.7) is shown in the gray region, that represents the values of prestresses that the mixture can admit. Moreover, the prestress is prescribed on the mixture and its partition between the solid and the fluid part can be chosen in a certain range, see e.g. Sciarra et al. (2001). We

\footnotetext{
3 We have already underlined that this condition is just an hypothesis that is not used in the paper. However, the graphics in Fig. 2 can be made only if we set the constitutive parameters to be constant with respect to prestress.

${ }^{4}$ Let us remark that the partial mass density of a constituent $\alpha$ is the mass of such species per unit volume of the mixture. Therefore, if the fluid is water with mass density equal to $1000 \mathrm{Kg} / \mathrm{m}^{3}$ and the volume fraction (the porosity) is 0.3 , then the (initial) partial mass density of the fluid must be $300 \mathrm{Kg} / \mathrm{m}^{3}$.
} 


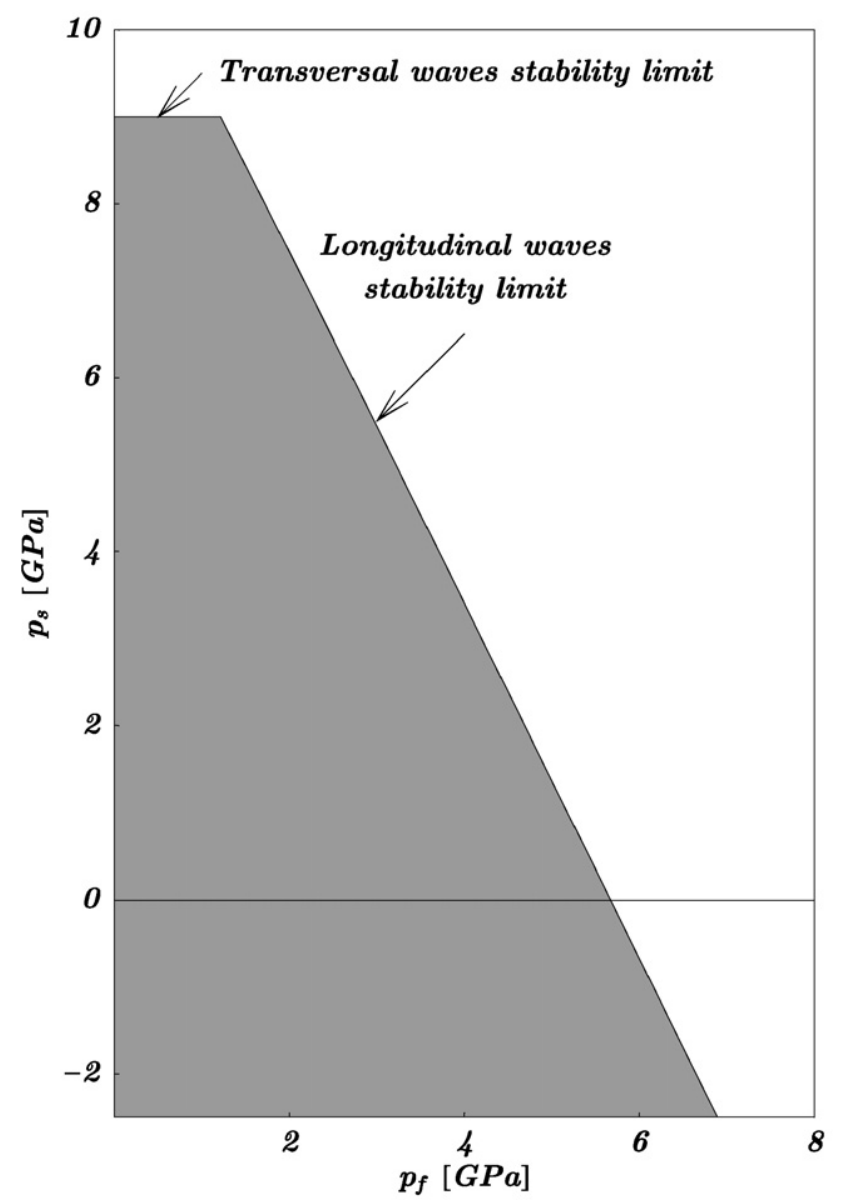

Fig. 2. Non-instability region is defined by the inequalities (5.4) $)_{2},(5.6)$ and (5.7), and it is shown here in gray. Numerical values of Eqs. (7.2) are used and only positive values of fluid pressure are shown. In the range of pressures that is represented in the graphic it is evident that the hyperbola can be approximated by a straight line. Let us remark that the origin of the axes, corresponding to a stress-free reference configuration, is included in such non-instability region.

adopt one of the possible criterion, that is a mass fraction criterion. If $p_{0}=0.5 \mathrm{GPa}$ is the value of the initial pressure prescribed on the mixture, then we choose the following numerical values,

$$
p_{0}=0.5 \mathrm{GPa} \quad p_{0}^{s}=\xi_{0}^{s} p \simeq 0.45 \mathrm{GPa}, \quad p_{0}^{f}=\xi_{0}^{f} p \simeq 0.05 \mathrm{GPa} .
$$

In these conditions we can also draw in Fig. 3, the phase velocities $V_{p h}=\operatorname{Re}(\omega / k)$ and the attenuation coefficients $\gamma=\operatorname{Im}(k)$ of longitudinal and transverse waves in terms of the real frequency $\omega$.

We underline that similar graphics are derived from the model of Wilmanski $(1996 ; 2000 ; 2004)$, in which the kinematical description is characterised not only with the placement of fluid and of solid components but also with the porosity field. This means that in order to obtain the dispersion relation of a porous medium saturated with fluid is not important to consider the balance of porosity, that is an equation difficult to interpret.

\subsection{A parametric analysis}

In order to better understand the effect of the initial stress $p_{0}$ and coupling coefficient $\gamma_{s f}$ on the propagation of monochromatic waves, a parametric analysis of the phase velocity and attenuation coefficient is developed. In particular we separately discuss the behaviour of longitudinal and shear waves in the following three cases: (i) pre-stress $p_{0^{-}}$ parametrisation (assuming $\gamma_{s f}=0$ ); (ii) coupling $\gamma_{s f}$-parametrisation (assuming $p_{0}=0$ ); (iii) no effective-coupling parametrisation $\left(\tilde{\gamma}_{s f}=0\right.$, which implies $\left.\gamma_{s f}=-2 p_{0} / \rho_{0}^{3}\right)$. From now on we adopt the nomenclature introduced by 

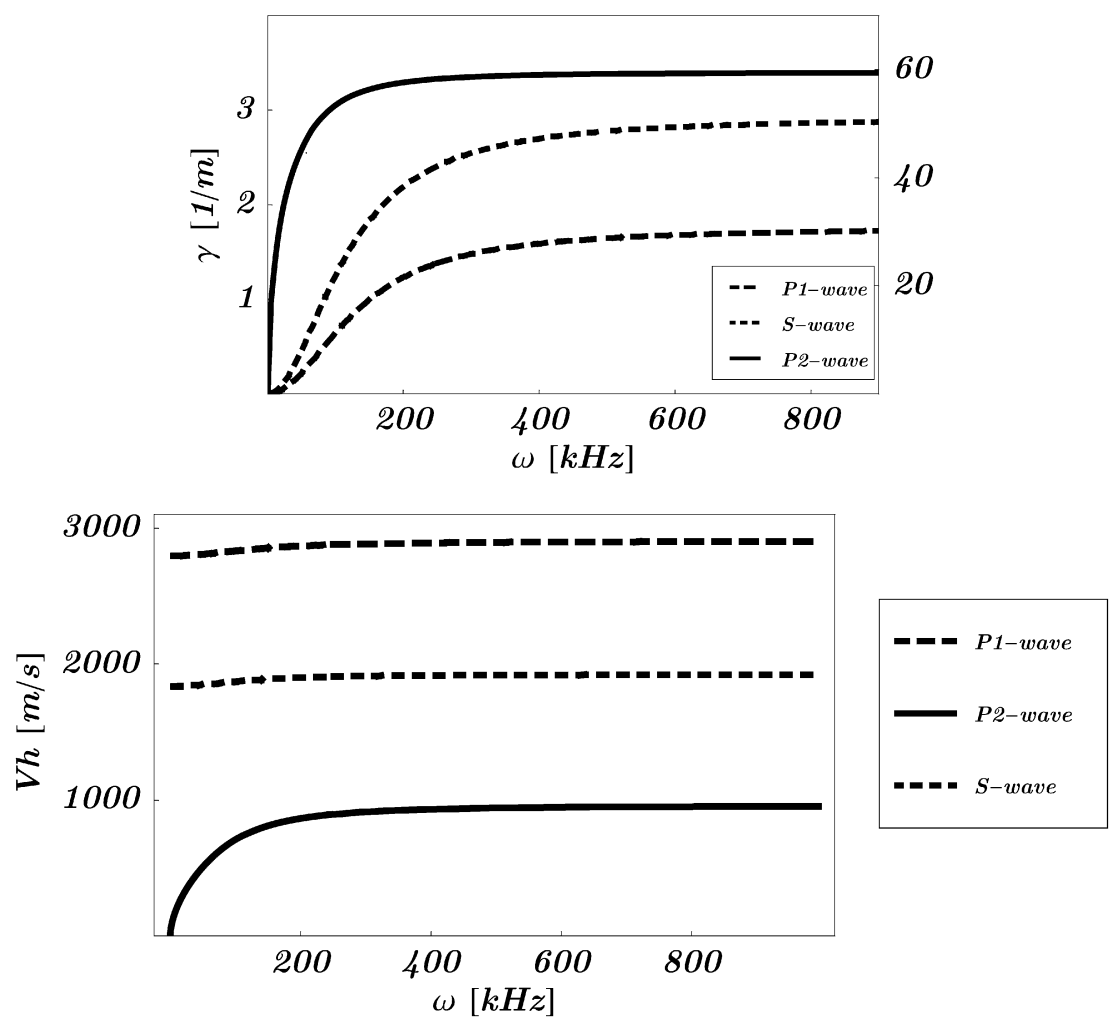

Fig. 3. Phase velocities $V_{h}$ and attenuation coefficients $\gamma$ of the $\mathrm{P} 1, \mathrm{P} 2$ and $\mathrm{S}$ waves. The $\mathrm{P} 1$ wave is generally considered to be the longitudinal wave that propagates through the solid component and the $\mathrm{P} 2$ wave is the analogous for the fluid. The $\mathrm{S}$ wave is, on the other hand, generally considered to be the transversal wave related to the solid. In our context all the waves do not propagate only through one component but through the mixture itself and the numerical values of phase velocities and attenuation coefficients depend upon the characteristics of all the components of the mixture, upon the pre-stress and the interaction parameters $D$ and $\gamma_{s f}$. We finally remark that the $\mathrm{P} 2$ wave is highly attenuated and this is the reason why it is very difficult, from an experimental point of view, to measure it.

Biot (see Biot, 1956a) in order to distinguish the two longitudinal waves, one from the other: the poorly attenuated wave is called P1 wave, which mainly propagates through the solid skeleton; the highly attenuated one is called P2 and it propagates essentially through the fluid.

$\mathrm{S}$-waves are not affected by the solid-fluid coupling $\gamma_{s f}$, which is correct bearing in mind that the fluid is not viscous. Therefore only case (i) is discussed in Fig. 4 . When increasing the initial stress $p_{0}$, the phase velocity becomes smaller and smaller, while the attenuation coefficient tends to infinity. Pre-stress can be increased until the effective stiffness $\mu-p_{s}^{0}$ becomes zero, that is the case of the so-called static solution, already discussed in Section 4.2 in the zero limit. We note that $\mu=p_{s}^{0}$ characterises the static solution even in classical elastic Cauchy continua.

For longitudinal waves we claim that the pre-stress parametrisation (i) in Figs. 5 and 6 and the $\gamma_{s f}$-parametrisation (ii) in Figs. 7 and 8 provide similar results. In Figs. 5 and 7 we analyse the so called P1 waves and in Figs. 6 and 8 we analyse the so called P2 waves. The phase velocity of the highly attenuated wave (the P2 wave) becomes smaller and smaller when the initial stress $p_{0}$ or the coupling coefficient $\gamma_{s f}$ grows up. This behaviour is exactly that of the P2 waves introduced by Biot (see Biot, 1956a).

P1 waves are characterised by a small value of the attenuation coefficient (which in particular tends to zero when increasing the coupling coefficient $\gamma_{s f}$ ) and a non-vanishing phase velocity, when limiting the analysis to the stability domain of Fig. 2.

Even in case (iii), when the initial stress $p_{0}$ is sufficiently close to zero, it is possible to detect a highly attenuated wave (i.e., P2 wave) and another one which is characterised by small values of the attenuation coefficient (i.e., P1 wave); however, when approaching the $p_{0}$ stability limit, the roles played by the two longitudinal waves are not so clear as in the previous cases. In particular, it is the wave poorly attenuated for small values of pre-stress which blows- 

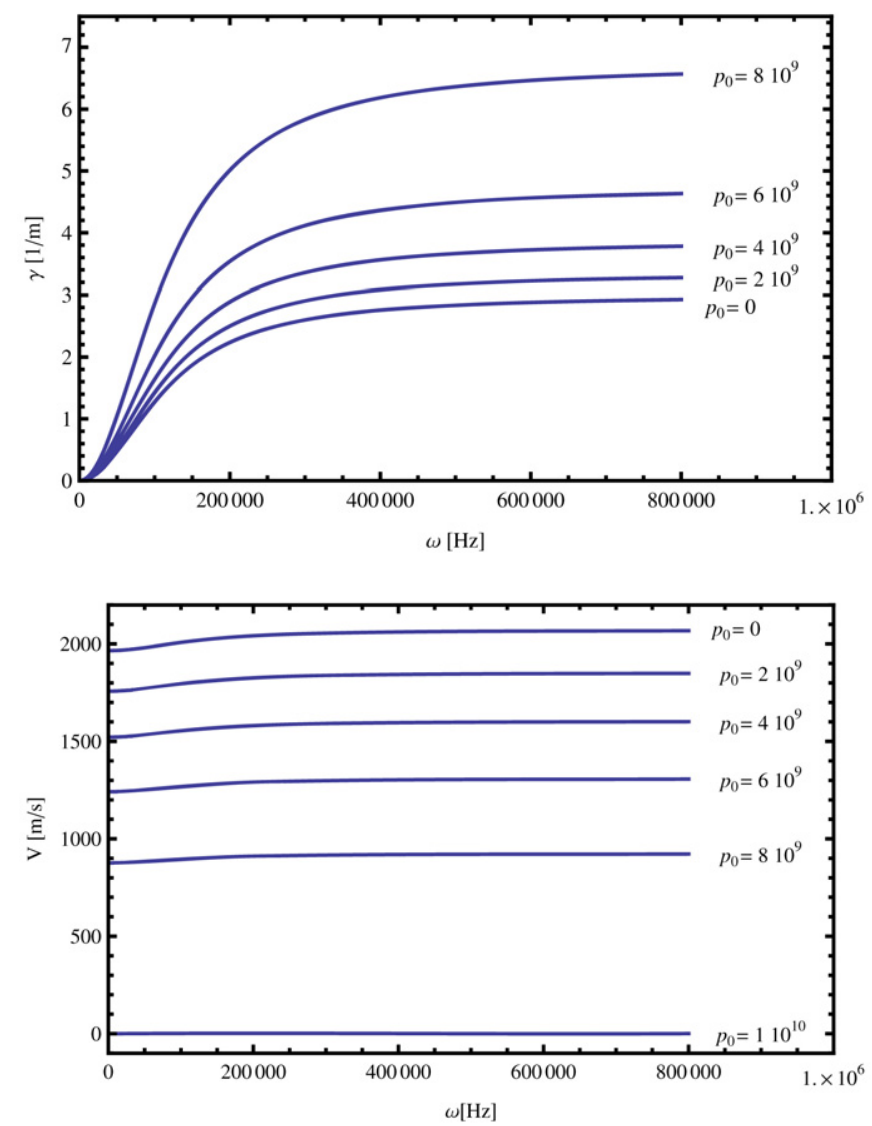

Fig. 4. Case (i): $\gamma_{s f}=0$. Attenuation coefficient and phase velocity are plotted for $\mathrm{S}$ waves and parameterised with $p_{0}$. Increasing $p_{0}$, the attenuation coefficient increases and the phase velocity decreases.

up when increasing $p_{0}$, while the amplitude of the highly attenuated wave does not decrease, see Figs. 9 and 10 . Thus, approaching the stability limit results in a more attenuated wave than the one associated to cases (i) and (ii).

\section{Conclusions}

From the kinematical point of view, the model derived in this paper is very simple, in the sense that only the placements of both components belong to the set of the state parameters of the mixture while the porosity is not taken into account. This is a simplification that does not modify the qualitative features of the dispersion relation as derived in the model of Wilmanski.

The governing equations are derived in this paper for a solid-fluid mixture and can be related to a porous medium saturated with a fluid, e.g., for a soil. They are deduced in a well-grounded variational context assuming a special form of the action functional and defining two families of variations. The set of field equations apparently depend on the prestress which is assumed not to be small and a set of restrictions on its values are derived so as to determine stability/instability conditions. This is a very important result, as a matter of facts the theory is capable to predict the stability conditions of the material under wave perturbations starting from the knowledge of the initial stress.

We finally remark that, in our derivation, the constitutive parameters need to be measured in a prestressed reference configuration, while we have assumed, in the numerical example, that these parameters coincide with those of a stressfree initial state. Such an approximation is generally assumed in the literature, but should be carefully checked in an experimental context. 

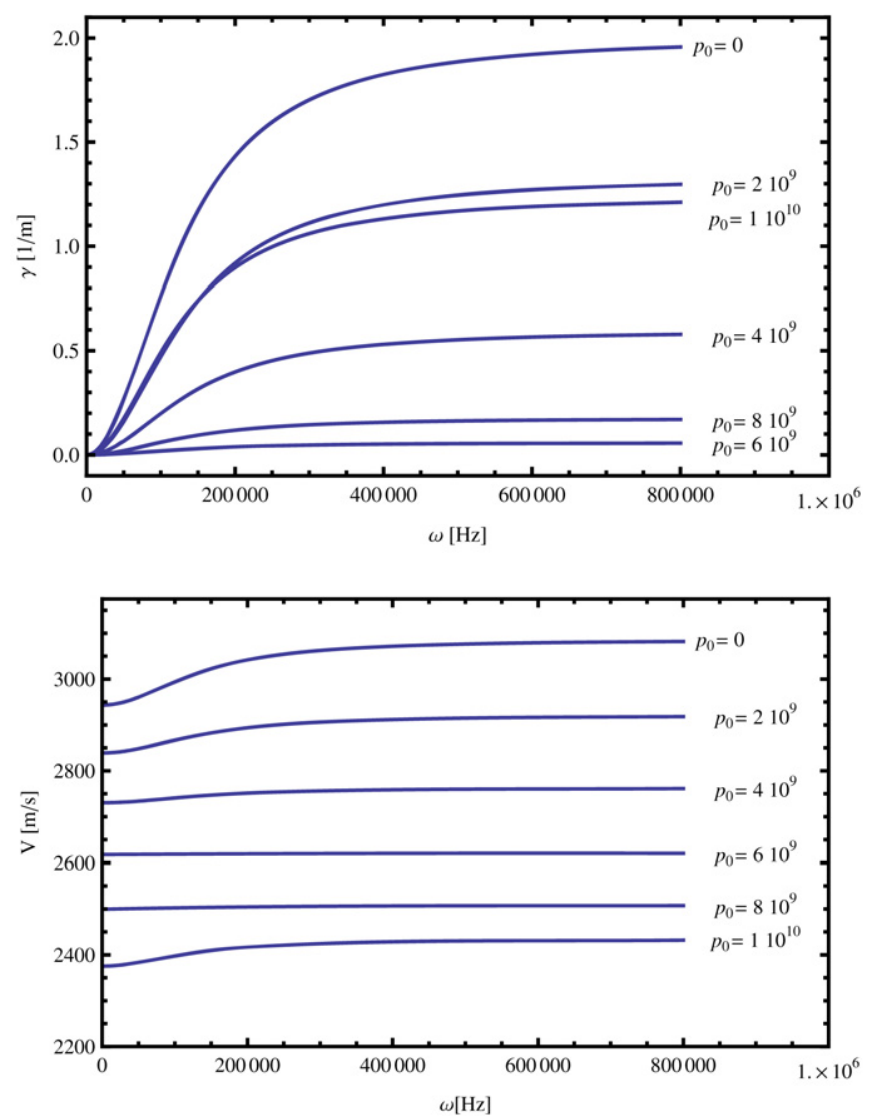

Fig. 5. Case (i): $\gamma_{s f}=0$. Attenuation coefficient and phase velocity are plotted for P1 waves and parameterised with $p_{0}$. Increasing $p_{0}$, the phase velocity increases and the attenuation coefficient initially decreases and than increases.

\section{Acknowledgement}

We acknowledge the financial support assured by the Universitá di Roma La Sapienza during the years 2004 (Ateneo-C26A045073), 2005 (Ateneo-C26A050779) and 2006 (Ateneo-C26A06KXKX).

\section{Appendix A. Solid and fluid variations}

The solid variation of the action is,

$$
\begin{aligned}
\delta_{s} \mathcal{A}= & \int_{t_{0}}^{t_{1}} \int_{\mathcal{B}_{s}}\left[\varepsilon J_{s}\left(\nabla_{\mathbf{x}} \cdot \eta_{s}\right) \mathcal{L}+J_{s} \delta_{s} \mathcal{L}\right]=\varepsilon \int_{t_{0}}^{t_{1}} \int_{\mathcal{B}_{t}}\left\{\nabla_{\mathbf{x}} \cdot \eta_{s}\left[\frac{1}{2} \varrho_{s}\left|\mathbf{v}_{s}\right|^{2}+\frac{1}{2} \varrho_{f}\left|\mathbf{v}_{f}\right|^{2}-\varrho \Psi\right]\right. \\
& -\frac{1}{2}\left(\varrho_{s} \nabla_{\mathbf{x}} \cdot \eta_{s}\right)\left|\mathbf{v}_{s}\right|^{2}+\varrho_{s} \mathbf{v}_{s} \cdot\left(\frac{d_{s} \eta_{s}}{d t}\right)+\frac{1}{2}\left(\left(\nabla_{\mathbf{x}} \varrho_{f}\right) \cdot \eta_{s}\right)\left|\mathbf{v}_{f}\right|^{2}+\varrho_{f} \mathbf{v}_{f} \cdot\left(\left(\nabla_{\mathbf{x}} \hat{\mathbf{v}}_{f}\right) \eta_{s}\right) \\
& \left.+\left(\varrho_{s} \nabla_{\mathbf{x}} \cdot \eta_{s}\right) \Psi-\left(\left(\nabla_{\mathbf{x}} \varrho_{f}\right) \cdot \eta_{s}\right) \Psi-\varrho\left(\frac{\partial \Psi}{\partial \varrho_{f}}\left[\left(\nabla_{\mathbf{x}} \varrho_{f}\right) \cdot \eta_{s}\right]+\frac{\partial \Psi}{\partial \mathbf{F}_{s}}:\left[\nabla_{\mathbf{x}_{s}} \eta_{s}\right]\right)\right\}
\end{aligned}
$$

In order to simplify such formula, we note that,

$$
\int_{t_{0}}^{t_{1}} \int_{\mathcal{B}_{t}} \varrho_{s} \frac{d_{s}}{d t}\left(\mathbf{v}_{s} \cdot \eta_{s}\right)=\int_{t_{0}}^{t_{1}} \int_{\mathcal{B}_{s}} \varrho_{s}^{*} \frac{d_{s}}{d t}\left(\mathbf{v}_{s} \cdot \eta_{s}\right)=\int_{t_{0}}^{t_{1}} \frac{d_{s}}{d t} \int_{\mathcal{B}_{s}} \varrho_{s}^{*}\left(\mathbf{v}_{s} \cdot \eta_{s}\right)=\left[\int_{\mathcal{B}_{s}} \varrho_{s}^{*}\left(\mathbf{v}_{s} \cdot \eta_{s}\right)\right]_{t=t_{0}}^{t=t_{1}}=0,
$$



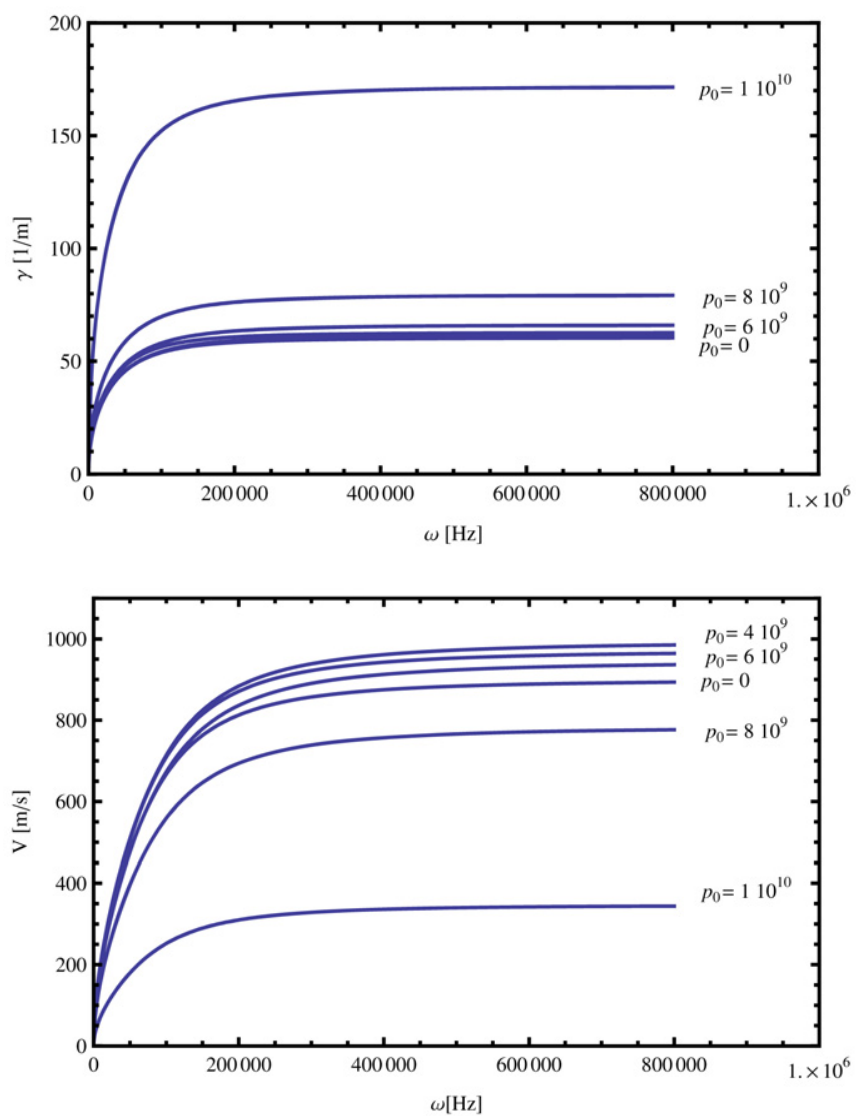

Fig. 6. Case (i): $\gamma_{s f}=0$. Attenuation coefficient and phase velocity are plotted for $\mathrm{P} 2$ waves and parameterised with $p_{0}$. Increasing $p_{0}$, the attenuation coefficient increases and the phase velocity initially increases and then decreases.

and therefore

$$
\int_{t_{0}}^{t_{1}} \int_{\mathcal{B}_{t}} \varrho_{s} \mathbf{v}_{s} \cdot\left(\frac{d_{s} \eta_{s}}{d t}\right)=\int_{t_{0}}^{t_{1}} \int_{\mathcal{B}_{t}} \varrho_{s} \frac{d_{s}}{d t}\left(\mathbf{v}_{s} \cdot \eta_{s}\right)-\int_{t_{0}}^{t_{1}} \int_{\mathcal{B}_{t}} \varrho_{s}\left(\frac{d_{s}}{d t} \mathbf{v}_{s}\right) \cdot \eta_{s}=-\int_{t_{0}}^{t_{1}} \int_{\mathcal{B}_{t}} \varrho_{s}\left(\frac{d_{s}}{d t} \mathbf{v}_{s}\right) \cdot \eta_{s} .
$$

We also know that,

$$
\begin{aligned}
& \nabla_{\mathbf{x}} \cdot\left(\frac{1}{2} \varrho_{f}\left|\mathbf{v}_{f}\right|^{2} \eta_{s}\right)=\frac{1}{2}\left(\nabla_{\mathbf{x}} \varrho_{f}\right) \cdot\left|\mathbf{v}_{f}\right|^{2} \eta_{s}+\varrho_{f} \mathbf{v}_{f} \cdot\left[\left(\nabla_{\mathbf{x}} \mathbf{v}_{f}\right) \eta_{s}\right]+\frac{1}{2} \varrho_{f}\left|\mathbf{v}_{f}\right|^{2} \nabla_{\mathbf{x}} \cdot \eta_{s}, \\
& \nabla_{\mathbf{x}} \Psi=\frac{\partial \Psi}{\partial \varrho_{f}} \nabla_{\mathbf{x}} \varrho_{f}+\frac{\partial \Psi}{\partial \mathbf{F}_{s}}:\left(\nabla_{\mathbf{x}} \mathbf{F}_{s}\right), \\
& \varrho \frac{\partial \Psi}{\partial \mathbf{F}_{s}}:\left[\left(\nabla_{\mathbf{x}} \eta_{s}\right) \mathbf{F}_{s}\right]=\nabla_{\mathbf{x}} \cdot\left[\eta_{s}\left(\varrho \frac{\partial \Psi}{\partial \mathbf{F}_{s}} \mathbf{F}_{s}^{T}\right)\right]-\eta_{s} \cdot\left[\nabla_{\mathbf{x}} \cdot\left(\varrho \frac{\partial \Psi}{\partial \mathbf{F}_{s}} \mathbf{F}_{s}^{T}\right)\right] .
\end{aligned}
$$

Thus,

$$
\begin{aligned}
\delta_{s} \mathcal{A}= & \varepsilon \int_{t_{0}}^{t_{1}} \int_{\mathcal{B}_{t}}-\varrho_{s}\left(\frac{d_{s}}{d t} \mathbf{v}_{s}\right) \cdot \eta_{s}+\nabla_{\mathbf{x}} \cdot\left(\frac{1}{2} \varrho_{f}\left|\mathbf{v}_{f}\right|^{2} \eta_{s}\right)-\left[\nabla_{\mathbf{x}} \cdot\left(\eta_{s} \varrho_{f} \Psi\right)\right] \\
& +\left[\varrho_{f} \frac{\partial \Psi}{\partial \mathbf{F}_{s}}:\left(\nabla_{\mathbf{x}} \mathbf{F}_{s}\right)-\varrho_{s} \frac{\partial \Psi}{\partial \varrho_{f}}\left(\nabla_{\mathbf{x}} \varrho_{f}\right)+\nabla_{\mathbf{x}} \cdot\left(\varrho \frac{\partial \Psi}{\partial \mathbf{F}_{s}} \mathbf{F}_{s}^{T}\right)\right] \cdot \eta_{s}-\varepsilon \int_{t_{0}} \int_{\partial \mathcal{B}_{t}}^{t_{1}}\left[\eta_{s}\left(\varrho \frac{\partial \Psi}{\partial \mathbf{F}_{s}} \mathbf{F}_{s}^{T}\right)\right] \cdot \mathbf{n},
\end{aligned}
$$



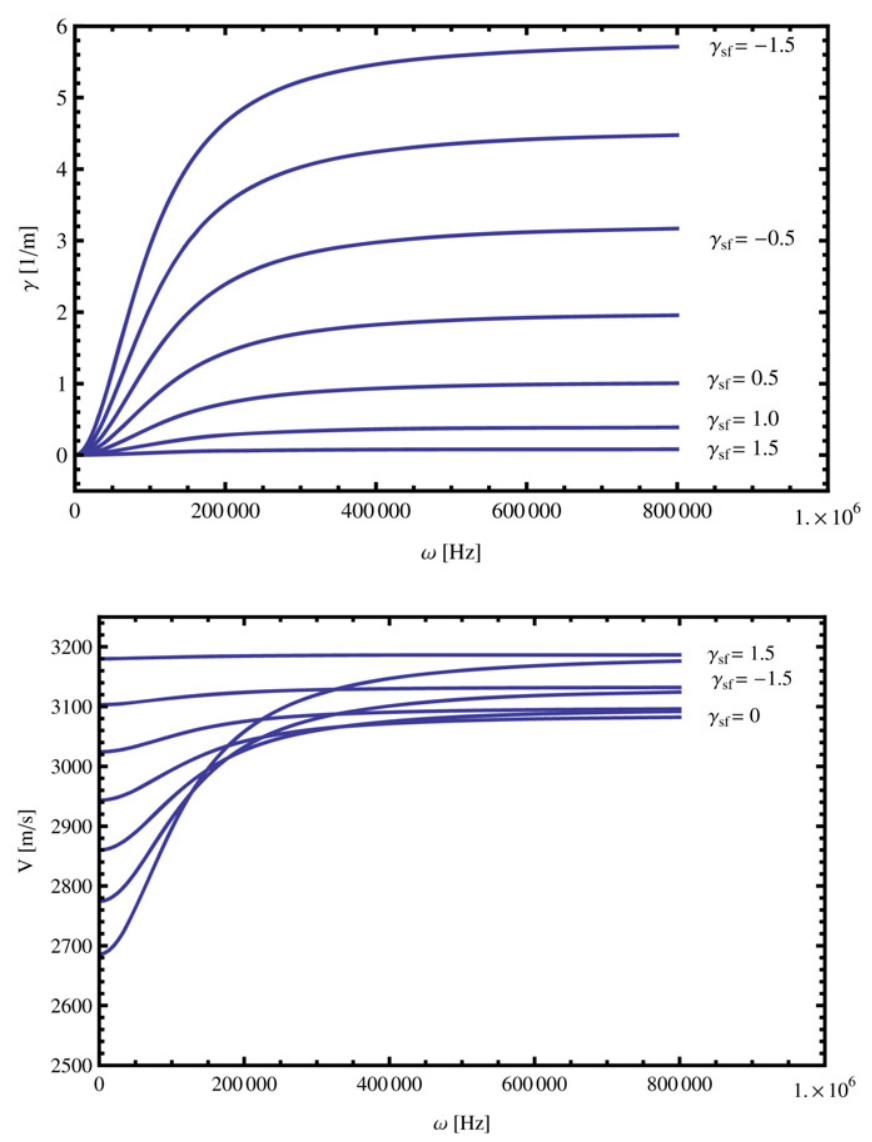

Fig. 7. Case (ii): $p_{0}=0$. Attenuation coefficient and phase velocity are plotted for P1 waves and parameterised with $\gamma_{s f}$. Increasing the value of $\gamma_{s f}$, the attenuation coefficient decreases.

that takes the (2.3) with $\alpha=s$. Analogously, the fluid variation of the action is,

$$
\begin{aligned}
\delta_{f} \mathcal{A}= & \varepsilon \int_{t_{0}}^{t_{1}} \int_{\mathcal{B}_{t}}-\varrho_{f}\left(\frac{d_{f}}{d t} \mathbf{v}_{f}\right) \cdot \eta_{f}+\nabla_{\mathbf{x}} \cdot\left(\frac{1}{2} \eta_{f} \varrho_{s}\left|\mathbf{v}_{s}\right|^{2}\right)-\left[\nabla_{\mathbf{x}} \cdot\left(\varrho_{s} \eta_{f} \Psi\right)\right] \\
& +\left[-\varrho_{f} \frac{\partial \Psi}{\partial \mathbf{F}_{s}}:\left(\nabla_{\mathbf{x}} \mathbf{F}_{s}\right)+\varrho_{s} \frac{\partial \Psi}{\partial \varrho_{f}} \nabla_{\mathbf{x}} \varrho_{f}-\nabla_{\mathbf{x}}\left(\varrho \varrho_{f} \frac{\partial \Psi}{\partial \varrho_{f}}\right)\right] \cdot \eta_{f}+\nabla_{\mathbf{x}} \cdot\left[\varrho_{f} \frac{\partial \Psi}{\partial \varrho_{f}} \eta_{f}\right],
\end{aligned}
$$

that takes the (2.3) with $\alpha=f$.

\section{Appendix B. Derivation of the solid Cauchy stress tensor}

The derivation of the solid Cauchy stress tensor is done in indicial notation. In such notation, the specific internal energy is,

$$
\begin{aligned}
\Psi= & \Psi_{0}+\left(\boldsymbol{\Psi}_{s}\right)_{a b}\left(\mathbf{E}_{s}\right)_{a b}+\frac{1}{2}\left(\boldsymbol{\Psi}_{s}\right)_{a b}\left(\mathbf{H}_{s}\right)_{c a}\left(\mathbf{H}_{s}\right)_{c b}+\Psi_{f} \tilde{\varrho}_{f} \\
& +\left(\boldsymbol{\Psi}_{s s}\right)_{a b c d}\left(\mathbf{E}_{s}\right)_{a b}\left(\mathbf{E}_{s}\right)_{c d}+\Psi_{f f} \tilde{\varrho}_{f}^{2}+\tilde{\varrho}_{f}\left(\boldsymbol{\Psi}_{s f}\right)_{a b}\left(\mathbf{E}_{s}\right)_{a b},
\end{aligned}
$$

so that

$$
\left(\frac{\partial \Psi}{\partial \mathbf{H}_{s}}\right)_{i j}=\frac{\partial \Psi}{\partial\left(\mathbf{H}_{s}\right)_{i j}}=\varrho_{0}\left(\boldsymbol{\Psi}_{s}\right)_{i j}+\varrho_{0} \tilde{\varrho}_{f}\left(\boldsymbol{\Psi}_{s f}\right)_{i j}+\varrho_{0}\left[\left(\mathbf{H}_{s}\right)_{i h}\left(\boldsymbol{\Psi}_{s}\right)_{h j}+\left(\boldsymbol{\Psi}_{s}\right)_{i h}\left(\mathbf{H}_{s}\right)_{j h}\right]
$$



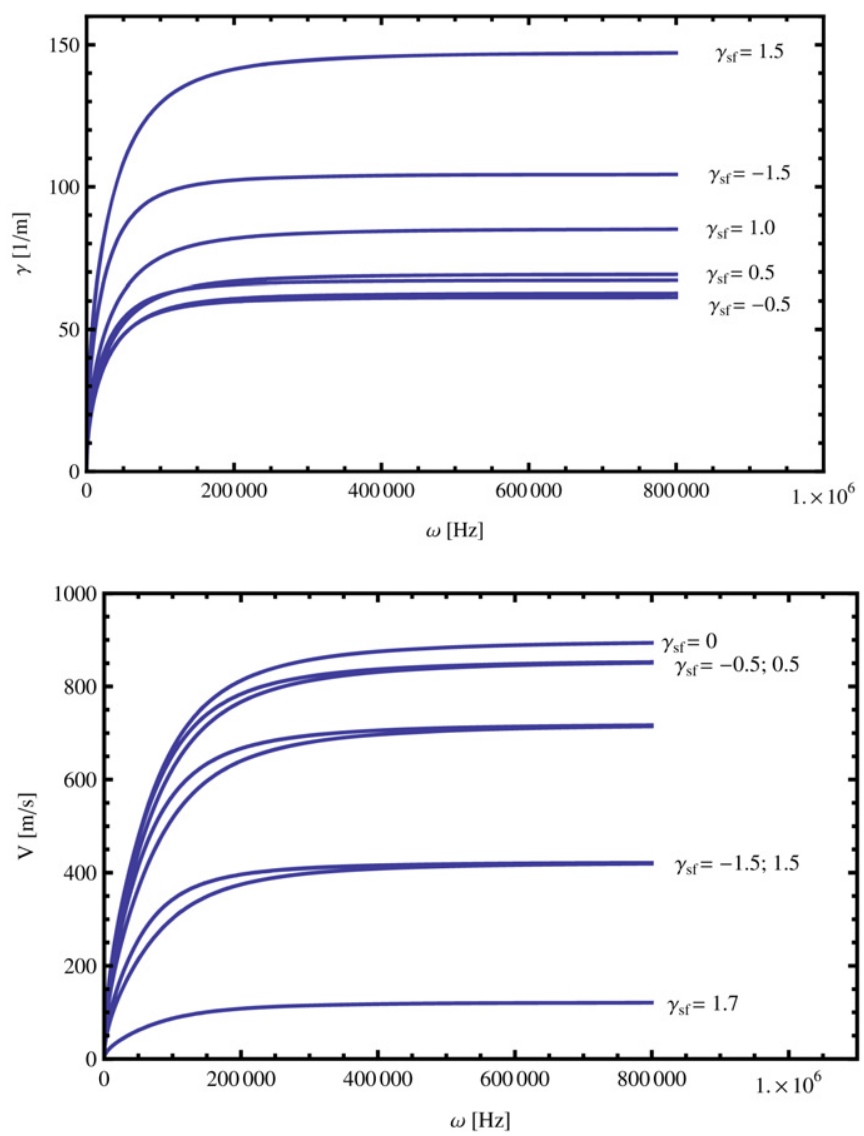

Fig. 8. Case (ii): $p_{0}=0$. Attenuation coefficient and phase velocity are plotted for P2 waves and parameterised with $\gamma_{s f}$. When approaching the stability limit for $\gamma_{s f}$ the attenuation coefficients tend to infinity and the phase velocity progressively decreases.
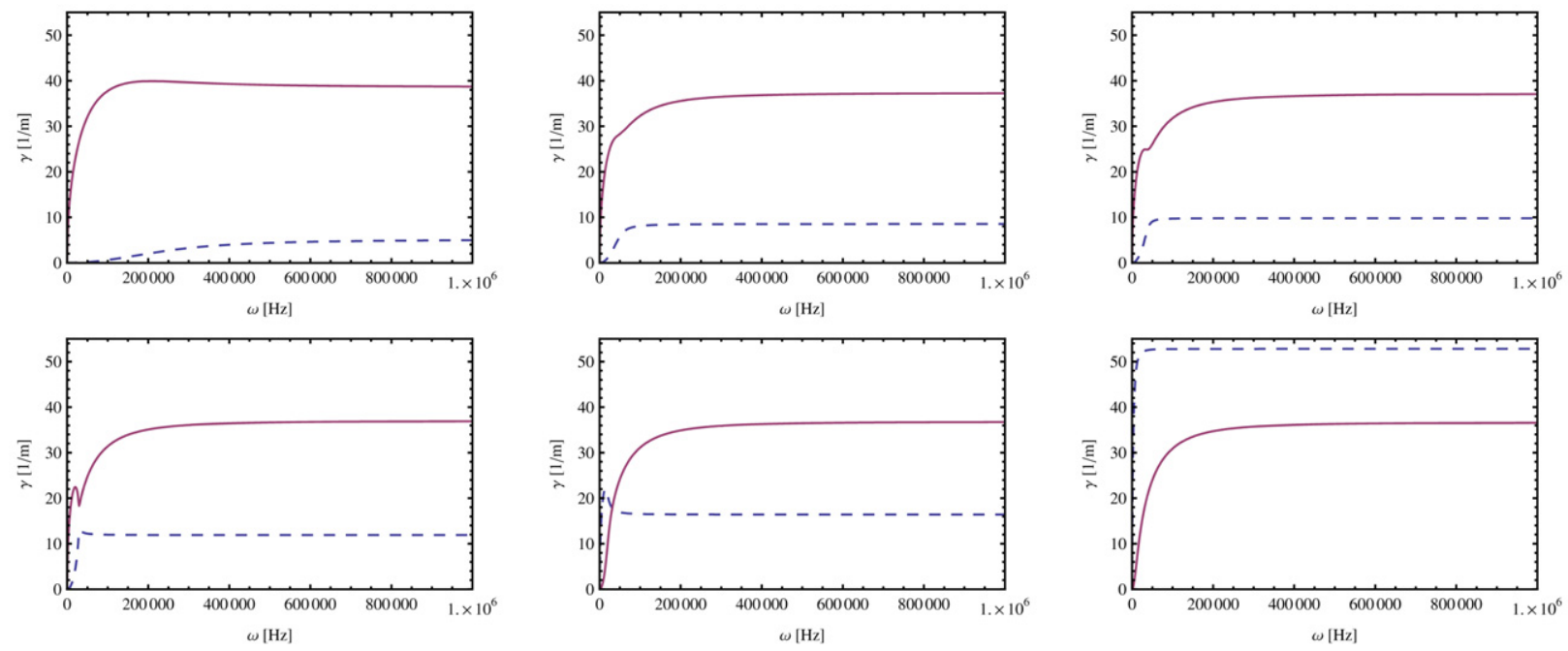

Fig. 9. Case (iii): $\tilde{\gamma}_{s f}=0$ and $\gamma_{s f}=-2 p_{0} / \rho_{0}^{3}$. Attenuation coefficient of $\mathrm{P} 1$ and $\mathrm{P} 2$ waves. The values of $p_{0}$ in the six graphics are, respectively, 14.90 GPa, 15.25 GPa, 15.30 GPa, 15.35 GPa, 15.40 GPa, 15.45 GPa. 

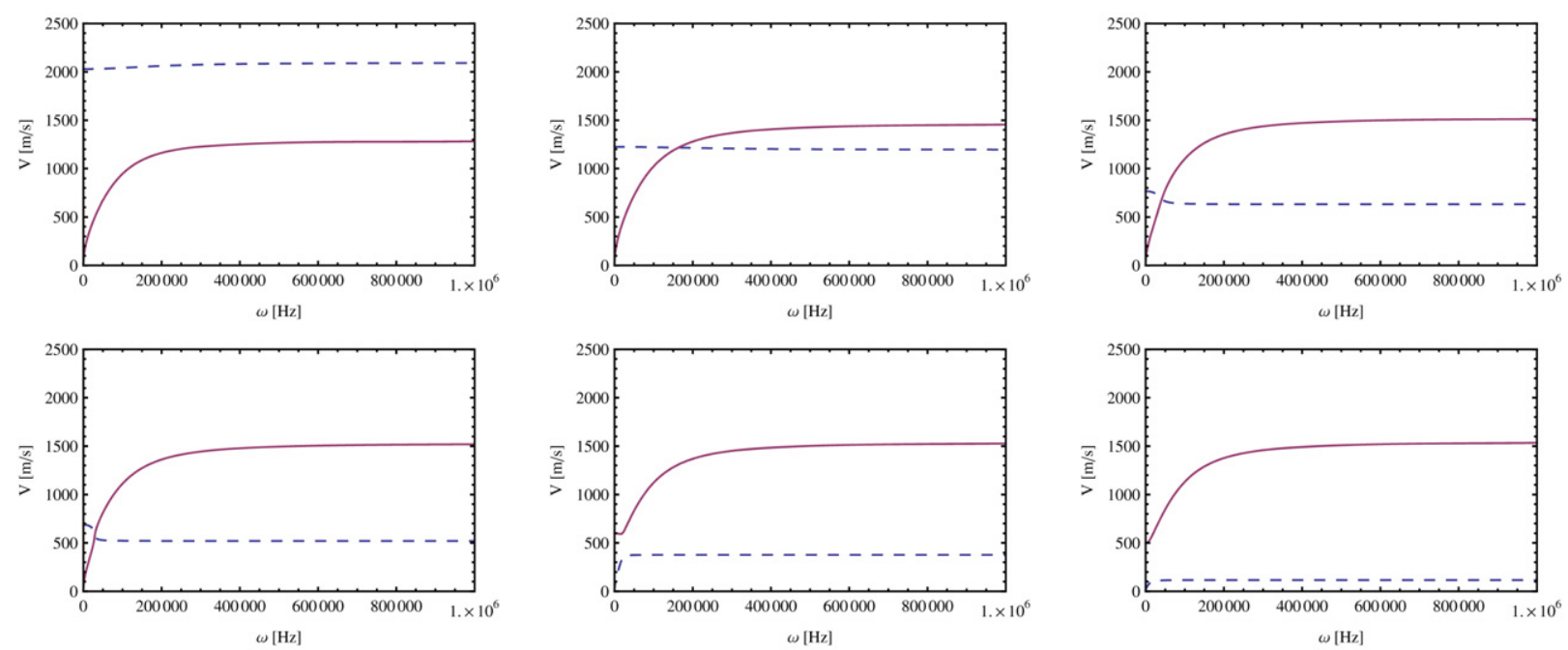

Fig. 10. Case (iii): $\tilde{\gamma}_{s f}=0$ and $\gamma_{s f}=-2 p_{0} / \rho_{0}^{3}$. Phase velocity of $\mathrm{P} 1$ and $\mathrm{P} 2$ waves. The values of $p_{0}$ in the six graphics are, respectively, 14.60 GPa, 14.90 GPa, 15.30 GPa, 15.35 GPa, 15.40 GPa, 15.45 GPa.

$$
+2 \varrho_{0}\left(\boldsymbol{\Psi}_{s s}\right)_{i j h k}\left(\mathbf{E}_{s}\right)_{h k}+\tilde{\varrho}_{f}\left(\boldsymbol{\Psi}_{s}\right)_{i j}-\varrho_{0}^{s}\left(\mathbf{H}_{s}\right)_{a a}\left(\boldsymbol{\Psi}_{s}\right)_{i j}
$$

and the (3.5) is achieved.

\section{References}

Abellan, M.A., de Borst, R., 2006. Wave propagation and localisation in a softening two-phase medium. Comput. Methods Appl. Mech. Engrg. 195 (1), 5011-5019.

Batra, G., Bedford, A., 1988. Acceleration waves in a fluid mixture with compressible constituents. Arch. Ration. Mech. Anal. 101 (1), 41-56.

Batra, G., Bedford, A., Drumheller, D.S., 1986. Applications of Hamilton's principle to continua with singular surfaces. Arch. Ration. Mech. Anal. 93 (3), 223-251.

Beak, S., Srinivasa, A.R., 2004. Diffusion of a fluid through an elastic solid undergoing large deformation. Int. J. Non-Linear Mech. 39, $201-218$. Bedford, A., Drumheller, D.S., 1978. A variational theory of immiscible mixtures. Arch. Ration. Mech. Anal. 68, 37-51.

Bedford, A., Drumheller, D.S., 1979. A variational theory of porous media. Int. J. Solids Structures 15, 967-980.

Bedford, A., Stern, M., 1972. Multi-continuum theory for composite elastic materials. Acta Mech. 14 (2-3), 85-102.

Benallal, A., Comi, C., 2003. Perturbation growth and localization in fluid-saturated inelastic porous media under quasi-static loadings. J. Mech. Phys. Solids 51, 851-899.

Biot, M.A., 1941. General theory of three-dimensional consolidation. J. Appl. Phys. 12, 155-164.

Biot, M.A., 1955. Theory of elasticity and consolidation for a porous anisotropic solid. J. Appl. Phys. 26, 182-185.

Biot, M.A., 1956a. Theory of propagation of elastic waves in a fluid-saturated porous solid I. Low-frequency range. J. Acoust. Soc. Am. 28, 168-178.

Biot, M.A., 1956b. General solutions of the equations of elasticity and consolidation for a porous material. J. Appl. Mech. 78, 91-96.

Biot, M.A., 1962. Mechanics of deformation and acoustic propagation in porous media. J. Appl. Phys. 33 (4), 1482-1498.

Biot, M.A., Willis, D.G., 1957. The elastic coefficients of the theory of consolidation. J. Appl. Mech. 24, 594-601.

Bluhm, J., de Boer, R., Wilmanski, K., 1995. The thermodynamic structure of the two-component model of porous incompressible materials with true mass density. Mech. Res. Com. 22 (2), 171-180.

Borrelli, A., Patria, M.C., 1984. Propagation of discontinuity waves of any order through an elastic solid saturated with an inviscid fluid. J. Appl. Math. Phys. 35, 373-386.

Bowen, R.M., 1982. Compressible porous media models by use of the theory of mixtures. Int. J. Engrg. Sci. 20 (6), 697-735.

Bowen, R.M., 1980. Incompressible porous media models by use of the theory of mixtures. Int. J. Engrg. Sci. 18, 1129-1148.

Bowen, R.M., 1976. Theory of mixtures. In: Eringen, A.C. (Ed.), Continuum Physics. III. Mixtures and Elastic Medium Field Theories. Academic Press, New York.

Bowen, R.M., Chen, P.J., 1975. Waves in a binary mixture of linear elastic materials. J. Mécanique 14 (2), 237-265.

Coussy, O., 2004. Poromechanics. Wiley.

Coussy, O., Dormieux, L., Detournay, E., 1998. From mixture theory to Biot's approach for porous media. Int. J. Solids Structures 35 (34-35), 4619-4635.

Cosenza, P., Ghoreychi, M., Bazargan-Sabet, B., de Marsily, G., 1999. In situ rock salt permeability measurement for long term safety assessment of storage. Int. J. Rock Mech. Min. 36, 509-526.

Courant, R., Hilbert, D., 1953. Methods of Mathematical Physics, vol. I. Interscience Publishers Inc., New York. 
Cowin, S.C., 1999. Bone poroelasticity. J. Biomech. 32 (3), 275-284.

de Boer, R., 1996. Highlights in the historical development of the porous media theory: Towards a consistent macroscopic theory. Appl. Mech. Rev. 49 (4), 201-262.

de Boer, R., 1998. The thermodynamic structure and constitutive equations for fluid-saturated compressible and incompressible elastic porous solids. Int. J. Solids Structures 35, 4557-4573.

de Boer, R., Ehlers, W., 1990. The development of the concept of effective stresses. Acta Meccanica 83, 77-92.

dell'Isola, F., Hutter, K., 1998a. A qualitative analysis of the dynamic of a sheared and pressurized layer of saturated soil. R. Soc. Lond. Ser. A Math. Phys. Eng. Sci. 454 (1980), 3105-3120.

dell'Isola, F., Hutter, K., 1998b. What are the dominant thermodynamical processes in the basal sediment layer of large ice sheets?. R. Soc. Lond Ser. A Math. Phys. Eng. Sci. 454 (1972), 1169-1195.

de Socio, L., Ianiro, N., Ponziani, D., 2003. Kinetic models for a gas filled porous matrix. Wave propagation. Transport in Porous Media 52, 95-109. Fichera, G., 1972. Existence theorems in elasticity. In: Handbuch der Physik, VIa/2. Springer, Heidelberg, pp. 347-389.

Fillunger, P., 1936. Erbdaumechanik. Selbst Verlag des Verfassers, Wien.

Foch, J., Ford, G.W., 1970. The dispersion of sound in monoatomic gases. De Boer, J., Uhlenbeck, G.E. (Eds.), Studies in Statistical Mechanics, vol. 5. North-Holland, Amsterdam.

Fu, Y.B., Ogden, R.W., 1999. Nonlinear stability analysis of prestressed elastic bodies. Continuum Mec. Thermodyn. 11 (3), $141-172$.

Gantmacher, F.R., 1960. The Theory of Matrices. Chelsea Publ. Company, New York.

Garg, S.K., Neyfeh, A.H., Good, A.J., 1974. Compressional waves in fluid-saturated elastic porous media. J. Appl. Phys. 45 (5), $1968-1974$.

Garg, S.K., Neyfeh, A.H., 1986. Compressional wave propagation in liquid and/or gas saturated elastic porous media. J. Appl. Phys. 60 (9), 3045-3055.

Gavrilyuk, S.L., 2005. Acoustic properties of a two-fluid compressible mixture with micro-inertia. Eur. J. Mech. B/Fluids 24, $397-406$.

Gavrilyuk, S.L., Gouin, H., 1999. A new form of governing equations of fluids arising from Hamilton's principle. Int. J. Engrg. Sci. 37, 1495-1520.

Gavrilyuk, S.L., Gouin, H., Perepechko, Yu.V., 1998. Hyperbolic models of homogeneous two-fluid mixtures. Meccanica 33, $161-175$.

Gouin, H., 1990. Variational theory of mixtures in continuum mechanics. Eur. J. Mech. B/Fluids 5, 469-491.

Gouin, H., Gavrilyuk, S.L., 1999. Hamilton's principle and Rankine-Hugoniot conditions for general motions of mixtures. Meccanica $34,39-47$.

Gouin, H., Ruggeri, T., 2003. Hamiltonian principle in the binary mixtures of Euler fluids with applications to the second sound phenomena. Rend. Mat. Acc. Lincei 14 (9), 69-83.

Hutter, K., Jönk, K., 2004. Continuum Methods of Physical Modelling, Continuum Mechanics, Dimensional Analysis, Turbulence. Springer.

Iesan, D., 1994. Prestressed Bodies. Longman Scientific \& Technical, John Wiley \& Sons, New York.

Liu, I.S., 2002. Continuum Mechanics. Springer, Berlin.

Müller, I., 1985. Thermodynamics. Pitman Advanced Publishing Program. Pitman, Boston.

Nigmatulin, R.I., Gubaidullin, A.A., 1992. Linear waves in saturated porous media. Transport in Porous Media 9, 135-142.

Placidi, L., Hutter, K., 2006. Thermodynamics of polycrystalline materials treated by the theory of mixtures with continuous diversity. Continuum Mech. Thermodyn. 17 (6), 409-451.

Pudasaini, S.P., Wang, Y., Hutter, K., 2005. Rapid motions of free-surface avalanches down curved and twisted channels and their numerical simulation. Phil. Trans. R. Soc. Lond. Ser. A Math. Phys. Eng. Sci. 363 (1832), 1551-1571.

Rajagopal, K.R., Tao, L., 1995. Mechanics of Mixture. Series on Advances in Mathematics for Applied Sciences, vol. 35. World Scientific Publishing Co., Inc., River Edge, NJ.

Rajagopal, K.R., Wieneman, A.S., Gandhi, M., 1986. On boundary conditions for a certain class of problems in mixture theory. Intern. J. Engrg. Sci. $24(8), 1453-1463$.

Sciarra, G., 2001. Modélisation de l'ecoulement d'un fluide dans une matrice solide déformable, PhD thesis.

Sciarra, G., dell'Isola, F., Hutter, K., 2001. A solid-fluid mixture model allowing for solid dilatation under external pressure. Continuum Mech. Thermodyn. 13, 287-306.

Sciarra, G., Hutter, K., Maugin, G.A., 2003. A variational approach to a micro-structured theory of solid-fluid mixtures. Arch. Appl. Mech. 73, 199-224.

Smeulders, D.M.J., De La Rosette, J.P.M., Van Dongen, M.E.H., 1992. Waves in partially saturated porous media. Transport in Porous Media 9, $25-37$.

Smeulders, D.M.J., Van Dongen, M.E.H., 1997. Wave propagation in porous media containing a dilute gas-liquid mixture: theory and experiments J. Fluid Mech. 343, 351-373.

Svendsen, B., 2001. On the Continuum modelling of materials with kinematic structure. Acta Meccanica 152, 49-80.

Terzaghi, K., 1925. Principles of soil mechanics. Eng. News-Records 95, 742-746, 796-800, 832-936, 974-978, 912-915, 1026-1029, 1064-1068.

Truesdell, C., 1957a. Sulle basi della termomeccanica. Nota I. Atti Accad. Naz. Lincei Rend. Cl. Sci. Mat. Nat. (8) XXII (I), 33-38.

Truesdell, C., 1957b. Sulle basi della termomeccanica. Nota II. Atti Accad. Naz. Lincei Rend. Cl. Sci. Mat. Nat. (8) XXII (I), $158-166$.

Truesdell, C., Toupin, R., 1960. The classical field theories. In: Flügge, S. (Ed.), Handbuch der Physik, III/1. Springer, Berlin, pp. $226-793$.

Truesdell, C., Noll, W., 1965. The nonlinear field theories of mechanics. In: Flügge, S. (Ed.), Handbuch der Physik, III/3. Springer, Berlin, pp. 1602.

Wang, Y., Hutter, K., Pudasaini, S.P., 2004. The Savage-Hutter theory: A system of partial differential equations for avalanches flows of snow, debris, and mud. Z. Angew. Math. Mech. 84 (8), 507-527.

Wilmanski, K., 2004. Elastic modelling of surface waves in single and multicomponent systems - Lecture Notes. CISM course, Udine.

Wilmanski, K., 2000. Mathematical Theory of Porous Media - Lecture Notes. XXV Summer School on Mathematical Physics, Ravello, September 2000.

Wilmanski, K., 1996. Porous media at finite strains. The new model with the balance equation for porosity. Arch. Mech. 48 (4), 591-628. 